\title{
Crystal graphs of higher level q-deformed Fock spaces, Lusztig $a$-values and Ariki-Koike algebras
}

\author{
Nicolas JACON *†
}

\begin{abstract}
We show that the different labelings of the crystal graph for irreducible highest weight $\mathcal{U}_{q}\left(\widehat{\mathfrak{s l}}_{e}\right)$-modules lead to different labelings of the simple modules for non semisimple Ariki-Koike algebras by using Lusztig $a$-values.
\end{abstract}

\section{Introduction}

The Ariki-Koike algebras have been introduced by Ariki and Koike in [AK. They can be seen as natural generalizations of Iwahori-Hecke algebras of type $A_{n-1}$ and $B_{n}$ and as special cases of cyclotomic Hecke algebras introduced by Broué and Malle in [BM]. Let $R$ be a commutative ring, let $l \in \mathbb{N}, n \in \mathbb{N}$ and let $v, x_{1}, x_{2}, \ldots, x_{l}$ be $l+1$ parameters in $R$. The Ariki-Koike algebra $\mathcal{H}_{R, n}:=\mathcal{H}_{R, n}\left(v ; x_{1}, \ldots, x_{l}\right)$ (or cyclotomic Hecke algebra of type $G(l, 1, n)$ ) over $R$ is the unital associative $R$-algebra presented by:

- generators: $T_{0}, T_{1}, \ldots, T_{n-1}$,

- relations:

$$
\begin{aligned}
& T_{0} T_{1} T_{0} T_{1}=T_{1} T_{0} T_{1} T_{0}, \\
& T_{i} T_{i+1} T_{i}=T_{i+1} T_{i} T_{i+1}(i=1, \ldots, n-2), \\
& T_{i} T_{j}=T_{j} T_{i}(|j-i|>1) \\
& \left(T_{0}-x_{1}\right)\left(T_{0}-x_{2}\right) \ldots\left(T_{0}-x_{l}\right)=0, \\
& \left(T_{i}-v\right)\left(T_{i}+1\right)=0(i=1, \ldots, n-1) .
\end{aligned}
$$

Assume that $R$ is a field of characteristic 0 . When $\mathcal{H}_{R, n}$ is a semisimple algebra, it is known that the simple $\mathcal{H}_{R, n}$-modules are given by the set of Specht modules $S \frac{\lambda}{R}$ parametrized by the $l$-partitions of rank $n$. Using results of Dipper and Mathas [DM], the non semisimple case can be reduced to the case where $R=\mathbb{C}$ and :

$$
v=\eta_{e}, \quad x_{i}=\eta_{e}^{v_{j}}, \quad j=1, \ldots, l,
$$

where $\eta_{e}:=\exp \left(\frac{2 i \pi}{e}\right) \in \mathbb{C}$ and where $0 \leq v_{1} \leq \ldots \leq v_{l}<e$ are positive integers.

\footnotetext{
* Laboratoire de Mathématiques Nicolas Oresme, Université de Caen

${ }^{\dagger}$ email: jacon@math.unicaen.fr
} 
In $\mathrm{Ac}$, Ariki has first provided a description of the simple modules in this modular case. Proving and generalizing a conjecture by Lascoux, Leclerc and Thibon, he has shown in Ad that the associated decomposition matrices can be identified with the Kashiwara-Lusztig canonical basis elements of the irreducible $\mathcal{U}\left(\widehat{\mathfrak{s l}}_{e}\right)$-module of highest weight $\Lambda_{v_{1}}+\Lambda_{v_{2}}+\ldots+\Lambda_{v_{l}}$ (where the $\Lambda_{i}$ denote the fundamental weights). It is known that these elements can be labeled by using the "crystal graph theory" and there are several ways to do that. Each of these ways corresponds to a certain realization of the Fock space as a module over the quantum group $\mathcal{U}_{q}\left(\widehat{\mathfrak{s l}}_{e}\right)$. In particular, one of these realizations leads to a labeling of the canonical basis elements by the "Kleshchev $l$-partitions" and Ariki has given a parametrization of the $\mathcal{H}_{\mathbb{C}, n}$-simple modules by using this class of $l$-partitions.

In [GR] and [G], Geck and Rouquier have given another approach for parametrizing the simple modules of the Hecke algebras of type $A_{n-1}$ and $B_{n}$ (which are special cases of Ariki-Koike algebras). This approach has been generalized to the case of Ariki-Koike algebras in Jp. Let $y$ be an indeterminate and let $A:=\mathbb{C}\left[y, y^{-1}\right]$. We consider the Ariki-Koike algebra $\mathcal{H}_{A, n}$ with the following choice of parameters:

$$
\begin{aligned}
& u_{j}=y^{l m^{(j)}} \eta_{l}^{j-1} \text { for } j=1, \ldots, l, \\
& v=y^{l} .
\end{aligned}
$$

where $\eta_{l}:=\exp \left(\frac{2 i \pi}{l}\right)$ and where for $j=1, \ldots, l$, we have $m^{(j)}=v_{j}-\frac{(j-1) e}{l}+\alpha e$ ( $\alpha$ is a positive integer such that $m^{(j)}>0$ for all $\left.j=1, \ldots, l\right)$.

If we specialize the indeterminate $y$ to $\eta_{l e}:=\exp \left(\frac{2 i \pi}{l e}\right)$ via a ring homomorphism $\theta$, we obtain the above Ariki-Koike algebra $\mathcal{H}_{\mathbb{C}, n}$. Let $K$ be the field of fractions of $A$ and let $\mathcal{H}_{K, n}:=K \otimes_{A} \mathcal{H}_{A, n}$. Then $\mathcal{H}_{K, n}$ is a split semisimple algebra and the simple $\mathcal{H}_{K, n}$-modules are the Specht modules $S_{K}^{\lambda}$ defined over $K$. We obtain a well-defined decomposition map $d$ between the Grothendieck groups of finitely generated $\mathcal{H}_{K, n}$-modules and $\mathcal{H}_{\mathbb{C}, n}$-modules. For $V \in \operatorname{Irr}\left(\mathcal{H}_{K, n}\right)$, we have equations:

$$
d([V])=\sum_{M \in \operatorname{Irr}\left(\mathcal{H}_{\mathbb{C}, n}\right)} d_{V, M}[M] .
$$

Using the theory of symmetric algebras, we can attach an integer $a(V)$ to each simple $\mathcal{H}_{K, n}$-module $V$. This is called the $a$-value of $V$ (for Hecke algebras of type $A_{n-1}$ and $B_{n}$, this value can also be defined using the Kazhdan-Lusztig theory). Then the main result of Jp asserts the following fact:

Theorem 1.1 For each $M \in \operatorname{Irr}\left(\mathcal{H}_{\mathbb{C}, n}\right)$, there exists a unique simple $\mathcal{H}_{K, n^{-}}$ module $V_{M}$ such that the following two conditions hold:

- $d_{V_{M}, M}=1$.

- if there exists $W \in \mathcal{H}_{K, n}$ such that $d_{W, M} \neq 0$ then $a(W)>a\left(V_{M}\right)$.

Moreover the application which sends $M$ to $V_{M}$ is injective. As a consequence the associated decomposition matrix is unitriangular and the following set is in natural bijection with $\operatorname{Irr}\left(\mathcal{H}_{\mathbb{C}, n}\right)$ :

$$
\mathcal{B}=\left\{V_{M} \mid M \in \operatorname{Irr}\left(\mathcal{H}_{\mathbb{C}, n}\right)\right\} .
$$


In Jp, using Ariki's theorem $\mathrm{Ad}$, we found that the set $\mathcal{B}$ is given by the Specht modules $S \frac{\lambda}{K}$ where $\underline{\lambda}$ runs over the set of "FLOTW $l$-partitions". This kind of multipartitions have been defined in [FL] and is another way to index the canonical basis elements of the associated $\mathcal{U}\left(\widehat{\mathfrak{s l}}_{e}\right)$-module.

Of course, the Ariki-Koike algebra $\mathcal{H}_{A, n}$ with the above choice of parameters is not the only algebra which specializes to $\mathcal{H}_{\mathbb{C}, n}$. In particular, assume that $f$ is an application from $\{1, \ldots, l\}$ to $\mathbb{N}$. We consider the Ariki-Koike algebra $\mathcal{H}_{A, n}^{f}$ with the following choice of parameters:

$$
\begin{aligned}
& u_{j}=y^{l m^{(j)}} \eta_{l}^{j-1} \text { for } j=1, \ldots, l, \\
& v=y^{l} .
\end{aligned}
$$

where for $j=1, \ldots, l$, we have $m^{(j)}=v_{j}-\frac{(j-1) e}{l}+\alpha e+f(j) e$ and $\alpha$ is a positive integer such that $m^{(j)}>0$ for all $j=1, \ldots, l$. Then the algebra $\mathcal{H}_{K, n}^{f}$ over $K:=\operatorname{Frac}(A)$ is still a split semisimple algebra but the $a$-values defined over each Specht module strongly depend on $f$. The aim of this paper is to show that Theorem 1.1 is true for all choices of $f$. Moreover, we show that in each case, the associated set $\mathcal{B}$ is parametrized by the crystal of the associated $\mathcal{U}_{q}\left(\widehat{\mathfrak{s l}}_{e}\right)$-module in one of the realizations of the Fock space mentionned above. The proof requires a combinatorial study of the deep results on higher level $q$-deformed Fock spaces proved by Uglov [U].

\section{Decomposition maps for Ariki-Koike algebras}

Let $R$ be a commutative associative ring with unit and let $v, x_{1}, \ldots, x_{l}$ be $l+$ 1 invertible elements in $R$. Let $n \in \mathbb{N}$. Let $\mathcal{H}_{R, n}:=\mathcal{H}_{R, n}\left(v ; x_{1}, \ldots, x_{l}\right)$ be the associated Ariki-Koike algebra as it is defined in the introduction. For a complete survey of the representation theory of $\mathcal{H}_{R, n}$, see [Ma].

It is known that this algebra is a "cellular" algebra in the sense of Graham and Lehrer GL and thus has "Specht modules" which are parametrized by the $l$-partitions of rank $n$. A $l$-partition $\underline{\lambda}$ of rank $n$ is a sequence of $l$ partitions $\underline{\lambda}=\left(\lambda^{(1)}, \ldots, \lambda^{(l)}\right)$ such that $\sum_{k=1}^{l}\left|\lambda^{(k)}\right|=n$. We denote by $\Pi_{l}^{n}$ the set of $l$ partitions of rank $n$.

For each $l$-partition $\underline{\lambda}$ of rank $n$, we can associate a remarkable $\mathcal{H}_{R, n}$-module $S \frac{\lambda}{R}$ which is free over $R$. This is called a Specht module (see the definition of "dual" Specht modules in DJM]). Assume that $R$ is a field. In general, the Specht modules are reducible and each of these modules can be endowed with a natural bilinear form. Let $\operatorname{rad}($.$) denotes the radical of this form. For \underline{\lambda} \in \Pi_{l}^{n}$, we denote :

$$
D \frac{\lambda}{R}:=S \frac{\lambda}{R} / \operatorname{rad}\left(S \frac{\lambda}{R}\right)
$$

Then the theory of cellular algebras gives the following result :

Theorem 2.1 (Graham-Lehrer GLL, Dipper-James-Mathas [DJM]) Assume that $R$ is a field then:

1. Non zero $D_{\bar{R}}^{\lambda}$ form a complete set of non-isomorphic simple $\mathcal{H}_{R, n}$-modules. Moreover, all of these modules are absolutely irreducible. 
2. If $\mathcal{H}_{R, n}$ is a semisimple algebra then it is split semisimple and for all $\underline{\lambda} \in \Pi_{l}^{n}$ we have $\operatorname{rad}\left(S \frac{\lambda}{R}\right)=0$. Thus, the $S \frac{\lambda}{R}$ form a complete set of non isomorphic simple modules.

Using this theorem, when $\mathcal{H}_{R, n}$ is semisimple, the simple modules are explicitely known and are given by the Specht modules. The following theorem gives a criterion of semisimplicty for the algebra $\mathcal{H}_{R, n}$.

Theorem 2.2 (Ariki $[A s]) \mathcal{H}_{R, n}$ is split semi-simple if and only if:

- for all $i \neq j$ and for all $d \in \mathbb{Z}$ such that $|d|<n$, we have:

$$
v^{d} x_{i} \neq x_{j},
$$

$$
\text { - } \prod_{i=1}^{n}\left(1+v+\ldots+v^{i-1}\right) \neq 0 \text {. }
$$

Hence we are reduced to understand the representations of $\mathcal{H}_{R, n}$ in the modular case. Assume that $R$ is a field of characteristic 0. Then, using the results of Dipper and Mathas $(\underline{\mathrm{DM}}])$ and Mathas $(\underline{\mathrm{Ms}})$, the non semisimple case can be reduced to the case where $R=\mathbb{C}$ and where all the $u_{i}$ are powers of the same root of unity $\eta_{e}:=\exp \left(\frac{2 i \pi}{e}\right)$ with $e \geq 2$. Let $\mathcal{H}_{\mathbb{C}, n}$ be the Ariki-Koike algebra over $\mathbb{C}$ with the following choice of parameters:

$$
\begin{aligned}
& x_{j}=\eta_{e}^{v_{j}} \text { for } j=1, \ldots, l, \\
& x=\eta_{e},
\end{aligned}
$$

where $0 \leq v_{l} \leq \ldots \leq v_{1}<e$. The problem of describing the simple modules of $\mathcal{H}_{\mathbb{C}, n}$ is linked with the problem of determining the decomposition map which we now define.

Let $\mathcal{H}_{A, n}$ be an Ariki-Koike defined over a commutative ring $A$ with unit. Let $K$ be the field of fractions of $A$. We assume that:

(1) $A$ is integrally closed in $K$,

(2) $\mathcal{H}_{K, n}:=K \otimes_{A} \mathcal{H}_{A, n}$ is split semi-simple,

(3) we have a ring homomorphism $\theta: A \rightarrow \mathbb{C}$ such that $\mathbb{C}=\operatorname{Frac}(\theta(A))$ and such that the specialized algebra $\mathbb{C} \otimes_{A} \mathcal{H}_{A, n}$ is the Ariki-Koike algebra $\mathcal{H}_{\mathbb{C}, n}$ with the above choice of parameters.

Then, by Gm], we have a well-defined decomposition map $d_{\theta}$ between the Grothendieck groups of finitely generated $\mathcal{H}_{K, n}$ and $\mathcal{H}_{\mathbb{C}, n}$-modules. In the context of cellular algebras, this application can be easily defined. Let:

$$
\Phi_{\left\{e ; v_{1}, \ldots, v_{l}\right\}}^{n}:=\left\{\underline{\lambda} \in \Pi_{l}^{n} \mid D_{\overline{\mathbb{C}}}^{\underline{\lambda}} \neq 0\right\} .
$$

Let $R_{0}\left(\mathcal{H}_{\mathbb{C}, n}\right)$ be the Grothendieck group of finitely generated $\mathcal{H}_{\mathbb{C}, n}$-modules. This is generated by the classes of simple $\mathcal{H}_{\mathbb{C}, n}$-modules $\left[D_{\mathbb{C}}\right]$ with $\underline{\lambda} \in \Pi_{l}^{n}$. Hence, for all $\underline{\lambda} \in \Pi_{l}^{n}$, there exist numbers $d_{\underline{\lambda}, \underline{\mu}}$ with $\underline{\mu} \in \Phi_{\left\{e ; v_{1}, \ldots, v_{l}\right\}}^{n}$ such that:

$$
\left[S \frac{\lambda}{\mathbb{C}}\right]=\sum_{\underline{\mu} \in \Phi_{\left\{e ; v_{1}, \ldots, v_{l}\right\}}^{n}} d_{\underline{\lambda}, \underline{\mu}}[D \underline{\underline{\underline{C}}}]
$$


The matrix $\left(d_{\underline{\lambda}, \underline{\mu}}\right)_{\underline{\lambda} \in \Pi_{l}^{n}, \underline{\mu} \in \Phi^{n}}$ is called the decomposition matrix of $\mathcal{H}_{\mathbb{C}, n}$ (where we denote $\left.\Phi^{n}:=\Phi_{\left\{e ; v_{1}, \ldots, v_{l}\right\}}^{n}\right)$. Let $\mathcal{F}_{n}$ be the $\mathbb{C}$-vectoriel space which is generated by the symbols $\llbracket S \underline{\lambda} \rrbracket$ with $\underline{\lambda} \in \Pi_{l}^{n}$. We obtain a homomorphism:

$$
\begin{array}{ll}
\mathcal{F}_{n} & \rightarrow R_{0}\left(\mathcal{H}_{\mathbb{C}, n}\right) \\
\llbracket S \underline{\lambda} \rrbracket & \mapsto\left[S_{\mathbb{C}}^{\underline{\lambda}}\right]=\sum_{\underline{\mu} \in \Phi^{n}} d_{\underline{\lambda}, \underline{\mu}}[D \underline{\mathbb{C}}] .
\end{array}
$$

Now, $\mathcal{F}_{n}$ can be naturally identified with the Grothendieck group of finitely generated modules over a semi-simple algebra $\mathcal{H}_{K, n}$ verifying $(1)-(3)$, by identifying the classes of simple $\mathcal{H}_{K, n}$-modules $\left[S_{K}^{\lambda}\right]$ with the symbols $\llbracket S \underline{\lambda} \rrbracket$. Hence, the decomposition map is defined as follows:

$$
\begin{aligned}
& d: \quad R_{0}\left(\mathcal{H}_{K, n}\right) \rightarrow R_{0}\left(\mathcal{H}_{\mathbb{C}, n}\right) \\
& {\left[S \frac{\lambda}{K}\right] \quad \mapsto \quad\left[S \frac{\lambda}{\mathbb{C}}\right]=\sum_{\underline{\mu} \in \Phi^{n}} d_{\underline{\lambda}, \underline{\mu}}\left[D \frac{\mu}{\mathbb{C}}\right] .}
\end{aligned}
$$

By Brauer Reciprocity, the decomposition matrix gives a description of the projective covers of the simple $\mathcal{H}_{\mathbb{C}, n}$-modules: for $\underline{\mu} \in \Phi_{\left\{e ; v_{1}, \ldots, v_{l}\right\}}^{n}$, the class of the projective cover $P \frac{\mu}{\mathbb{C}}$ of $D \frac{\mu}{\mathbb{C}}$ is given by:

$$
\left[P_{\overline{\mathbb{C}}}^{\underline{\mu}}\right]=\sum_{\underline{\lambda} \in \Pi_{l}^{n}} d_{\underline{\lambda}, \underline{\mu}}[S \underline{\underline{\mathbb{C}}}] .
$$

By results of Ariki and Uglov, the problem of determining the decomposition matrices for Ariki-Koike algebras can be translated to the problem of computing the canonical basis of $q$-deformed Fock spaces. In the next section, we recall these results.

\section{Canonical basis of higher level $q$-deformed Fock spaces}

The higher $q$-deformed Fock spaces have been introduced in [JM]. These spaces which are spanned by the set of "multipartitions" can be endowed with a structure of integrable $\mathcal{U}_{q}\left(\widehat{s l}_{e}\right)$-module. In [TU], generalizing works by Lascoux and Leclerc [LT], Takemura and Uglov have given a construction of canonical bases for these spaces. In this part, we review this construction following [U] (see also Us $)$. Then, we explain the links with the representation theory of Ariki-Koike algebras which are given by Ariki's theorem.

\section{A $q$-wedge products and $q$-deformed Fock spaces}

Let $q$ and $z$ be indeterminates and let $l$ and $e$ be positive integers. Let $V_{e}$ be an $e$-dimensional vector space over $\mathbb{Q}(q)$ with basis $v_{1}^{(e)}, v_{2}^{(e)}, \ldots, v_{e}^{(e)}$. We put $V_{e, l}:=\left(V_{e} \otimes V_{l}\right)\left[z, z^{-1}\right]$. For $a \in\{1, \ldots, e\}, b \in\{1, \ldots, l\}$ and $m \in \mathbb{Z}$, we put $k:=a+e(b-1)-e l m$ and $u_{k}:=v_{a}^{(e)} z^{m} v_{b}^{(l)}$. Then $V_{e, l}$ is a $\mathbb{Q}(q)$ vectoriel space with basis $\left\{u_{k} \mid k \in \mathbb{Z}\right\}$.

The $q$-wedge square $\bigwedge^{2} V_{e, l}$ of $V_{e, l}$ can be viewed as a $q$-deformation of the exterior square of $V_{e, l}$. This is a $\mathbb{Q}(q)$-vector space generated by the monomials $u_{k_{1}} \wedge u_{k_{2}}$ with $\left(k_{1}, k_{2}\right) \in \mathbb{Z}^{2}$. A basis of this space is given by the "ordered" 
monomials that is the monomials $u_{k_{1}} \wedge u_{k_{2}}$ such that $k_{1}>k_{2}$. Any monomial $u_{k_{1}} \wedge u_{k_{2}}$ can be expressed as a linear combination of ordered monomials using the following rules $(R 1),(R 2),(R 3)$ and $(R 4)$.

Let $k_{1} \leq k_{2}$ and for $i=1,2$, put $k_{i}=a_{i}+e\left(l-b_{i}\right)-e l m_{i}$ where $a_{i} \in$ $\{1, \ldots, e\}, b_{i} \in\{1, \ldots, l\}$ and $m_{i} \in \mathbb{Z}$. We define $\alpha:=\left(a_{2}-a_{1}\right)(\bmod e l)$ and $\beta:=\left(e\left(b_{1}-b_{2}\right)\right)(\bmod e l)$. Then the relations $(R 1),(R 2),(R 3)$ and $(R 4)$ are given as follows:

(R1) if $\alpha=0$ and $\beta=0$ :

$$
u_{k_{1}} \wedge u_{k_{2}}=-u_{k_{2}} \wedge u_{k_{1}}
$$

(R2) if $\alpha \neq 0$ and $\beta=0$ :

$$
\begin{aligned}
u_{k_{1}} \wedge u_{k_{2}}= & -q^{-1} u_{k_{2}} \wedge u_{k_{1}}+ \\
& +\left(q^{-2}-1\right) \sum_{m \geq 0} q^{-2 m} u_{k_{2}-\alpha-e l m} \wedge u_{k_{1}+\alpha+e l m}- \\
& -\left(q^{-2}-1\right) \sum_{m \geq 1} q^{-2 m+1} u_{k_{2}-\text { elm }} \wedge u_{k_{1}+e l m}
\end{aligned}
$$

(R3) if $\alpha=0$ and $\beta \neq 0$ :

$$
\begin{aligned}
u_{k_{1}} \wedge u_{k_{2}}= & q u_{k_{2}} \wedge u_{k_{1}}+ \\
& +\left(q^{2}-1\right) \sum_{m \geq 0} q^{2 m} u_{k_{2}-\beta-e l m} \wedge u_{k_{1}+\beta+e l m}+ \\
& +\left(q^{2}-1\right) \sum_{m \geq 1} q^{2 m-1} u_{k_{2}-\text { elm }} \wedge u_{k_{1}+e l m}
\end{aligned}
$$

(R4) if $\alpha \neq 0$ and $\beta \neq 0$ :

$$
\begin{aligned}
u_{k_{1}} \wedge u_{k_{2}}= & u_{k_{2}} \wedge u_{k_{1}}+ \\
& +\left(q-q^{-1}\right) \sum_{m \geq 0} \frac{q^{2 m+1}+q^{-2 m-1}}{q+q^{-1}} u_{k_{2}-\beta-e l m} \wedge u_{k_{1}+\beta+e l m}+ \\
& +\left(q-q^{-1}\right) \sum_{m \geq 0} \frac{q^{2 m+1}+q^{-2 m-1}}{q+q^{-1}} u_{k_{2}-\alpha-e l m} \wedge u_{k_{1}+\alpha+e l m}+ \\
& +\left(q-q^{-1}\right) \sum_{m \geq 0} \frac{q^{2 m}-q^{-2 m}}{q+q^{-1}} u_{k_{2}-\beta-\alpha-e l m} \wedge u_{k_{1}+\beta+\alpha+e l m}+ \\
& +\left(q-q^{-1}\right) \sum_{m \geq 1} \frac{q^{2 m}-q^{-2 m}}{q+q^{-1}} u_{k_{2}-\text { elm }} \wedge u_{k_{1}+e l m} .
\end{aligned}
$$

where the summations are taken over the set of ordered monomials.

For any integer $r \geq 2$, we can now define the $r$-fold $q$-wedge product $\bigwedge^{r} V_{e, l}$. This is the $\mathbb{Q}(q)$-vector space generated by the elements $u_{k_{1}} \wedge u_{k_{2}} \wedge \ldots \wedge u_{k_{r}}$ with $k_{i} \in \mathbb{Z}$. Again the ordered monomials that is the monomials $u_{k_{1}} \wedge u_{k_{2}} \wedge \ldots \wedge u_{k_{r}}$ with $k_{1}>k_{2}>\ldots>k_{r}$, form a basis of $\bigwedge^{r} V_{e, l}$. Moreover, an arbitrary monomial can be expressed as a linear combinaison of ordered monomials using the relations $(R 1),(R 2),(R 3)$ and $(R 4)$ in every adjacent pair of the factors.

Finally, for $s \in \mathbb{Z}$, the semi-infinite $q$-wedge product $\bigwedge^{s+\frac{\infty}{2}} V_{e, l}$ of charge $s$ is the inductive limit of $\bigwedge^{r} V_{e, l}$ where the maps $\bigwedge^{r} V_{e, l} \rightarrow \bigwedge^{r+1} V_{e, l}$ are given 
by $v \mapsto v \wedge u_{s-r}$. Hence, $\bigwedge^{s+\frac{\infty}{2}} V_{e, l}$ is the $\mathbb{Q}(q)$-vector space generated by the semi-infinite monomials $u_{k_{1}} \wedge u_{k_{2}} \wedge \ldots$ with $k_{i} \in \mathbb{Z}$ and such that $k_{i}=s-i+1$ for $i>>0$. A basis is given by the ordered monomials that is the monomials $u_{k_{1}} \wedge u_{k_{2}} \wedge \ldots$ with $k_{1}>k_{2}>\ldots$ and the relations $(R 1)-(R 4)$ provide a way to express an arbitrary monomial as a linear combinaison of ordered monomials.

Now, each semi-infinite ordered monomial can be labeled by a pair $\left(\underline{\lambda}, \mathbf{s}_{l}\right)$ where $\underline{\lambda} \in \Pi_{l}^{n}$ is a $l$-partition of rank $n$ and $\mathbf{s}_{l}=\left(s_{1}, \ldots, s_{l}\right) \in \mathbb{Z}^{l}$ is such that $\sum_{i=1}^{l} s_{i}=s$. This labeling coincides with that of [U] up to transformation $\left(s_{1}, \ldots, s_{l}\right) \mapsto\left(s_{l}, \ldots, s_{1}\right)$ and $\left(\lambda^{(1)}, \ldots, \lambda^{(l)}\right) \mapsto\left(\lambda^{(l)}, \ldots, \lambda^{(1)}\right)$. Let $u_{k_{1}} \wedge u_{k_{2}} \wedge$ $\ldots \in \bigwedge^{s+\frac{\infty}{2}} V_{e, l}$ be a semi-infinite ordered monomial, for $i=1,2, \ldots$, we put $k_{i}=a_{i}+e\left(l-b_{i}\right)-e l m_{i}$ where $a_{i} \in\{1, \ldots, e\}, b_{i} \in\{1, \ldots, l\}$ and $m_{i} \in \mathbb{Z}$. For $b=1, \ldots, l$, let $k_{1}^{(b)}>k_{2}^{(b)}>\ldots$ be the semi-infinite sequence obtained by ordering the elements of the set $\left\{a_{i}-e m_{i} \mid b_{i}=b\right\}$ in strictly decreasing order. Then, there is a unique $s_{b} \in \mathbb{Z}$ such that $k_{i}^{(b)}=s_{b}-i+1$ for $i>>1$. We put $\underline{\lambda}=\left(\lambda^{(1)}, \ldots, \lambda^{(l)}\right)$ where for $i>0, \lambda_{i}^{(b)}=k_{i}^{(b)}-s_{b}+i-1$ and $\mathbf{s}_{l}=\left(s_{1}, \ldots, s_{l}\right)$. This defines a bijection between the set of semi-infinite ordered monomials and the set of pairs $\left(\underline{\lambda}, \mathbf{s}_{l}\right)$ where $\underline{\lambda} \in \Pi_{l}^{n}$ and $\mathbf{s}_{l}=\left(s_{1}, \ldots, s_{l}\right) \in \mathbb{Z}^{l}$ is such that $\sum_{i=1}^{l} s_{i}=s$.

This bijection can be described as follows. First, the infinite decreasing sequence $\left(k_{1}, k_{2}, \ldots\right)$ can be pictured as a set of colored beads on an infinite runner. For example, the sequence $\underline{k}=(15,12,8,7,3,1,-2,-4,-5,-6, \ldots)$ is represented by the following abacus:

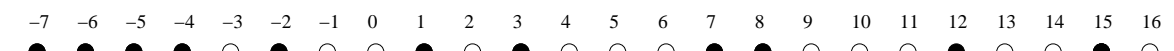

On the other hand, using the decomposition $k_{i}=a_{i}+e\left(l-b_{i}\right)-e l m_{i}$, the same sequence can be represented by an $l$-abacus, that is, as a set of colored beads on $l$ infinite runners. For $e=4$ and $l=3$, we obtain:

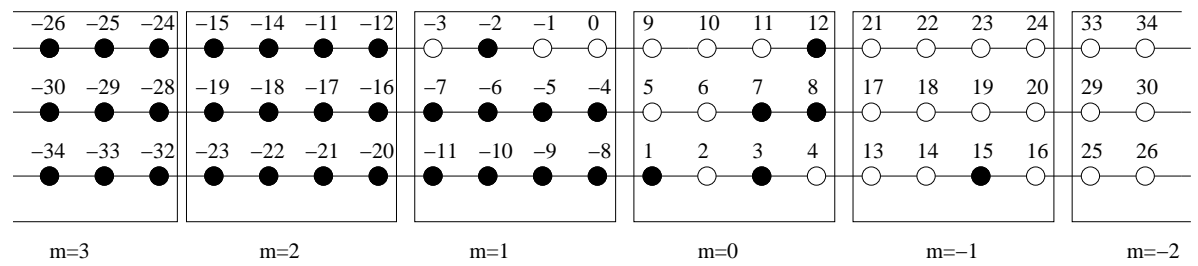

In the above representation, the colored beads are labeled by the integers $k_{i}$ (with $i=1,2, \ldots$ ). We can alternatively labeled them by the integers $k_{i}^{(b)}$ (with $i=1,2, \ldots$ and $b=1,2, \ldots, l)$ as follows:

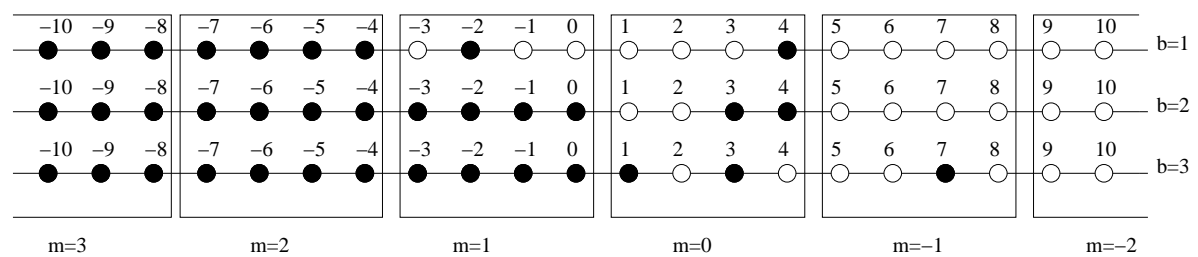

Let $\underline{\lambda}$ be the $l$-partition and $\mathbf{s}_{l}=\left(s_{1}, \ldots, s_{l}\right)$ be the $l$-tuple of integers associated to $\underline{k}$. For $i=1,2, \ldots$ and $b=1,2, \ldots, l$, we have $\lambda_{i}^{(b)}=k_{i}^{(b)}-s_{b}+i-1$. 
Thus, one can now easily determine $\underline{\lambda}$ by counting the number of non colored beads at the left of each colored bead on each runner. Continuing the above example, we obtain $\underline{\lambda}=((6,1),(2,2),(4,1))$ and $\mathbf{s}_{l}=(-2,2,3)$.

Now, the higher level $q$-deformed Fock space is defined to be the $\mathbb{C}(q)$-vector space generated by the symbols $\left|\underline{\lambda}, \mathbf{s}_{l}\right\rangle$ with $\underline{\lambda} \in \Pi_{l}^{n}$ :

$$
\mathcal{F}_{e, \mathbf{s}_{l}}:=\bigoplus_{\substack{\lambda \in \Pi_{l}^{n} \\ n \in \mathbb{N}}} \mathbb{C}(q)\left|\underline{\lambda}, \mathbf{s}_{l}\right\rangle .
$$

Thus, if we identify the semi-infinite ordered monomial $u_{\underline{k}} \in \bigwedge^{s+\frac{\infty}{2}} V_{e, l}$ with the pair $\left|\underline{\lambda}, \mathbf{s}_{l}\right\rangle$ defined by the above bijection, we get:

$$
\bigwedge^{s+\frac{\infty}{2}} V_{e, l}=\bigoplus_{s_{1}+\ldots+s_{l}=s} \mathcal{F}_{e, \mathbf{s}_{l}} .
$$

\section{B Canonical basis and Ariki's theorem}

Let $\mathcal{U}_{q}^{\prime}\left(\widehat{\mathfrak{s l}}_{e}\right)$ be the quantum group associated to the Lie algebra $\widehat{\mathfrak{s}}_{e}^{\prime}$. We denote by $e_{i}, f_{i}$ and $k_{i}$ with $i=0, \ldots, e-1$, the Chevalley generators. We can construct a structure of $\mathcal{U}_{q}^{\prime}\left(\widehat{\mathfrak{s l}}_{e}\right)$-module on $\bigwedge^{s+\frac{\infty}{2}} V_{e, l}$ (see [U] $\left.\S 3.5, \S 4.2\right]$ ) such that each of the subspace $\mathcal{F}_{e, \mathbf{s}_{l}}$ is stable with respect to this action. Before describing the action of the $\mathcal{U}_{q}^{\prime}\left(\widehat{\mathfrak{s l}}_{e}\right)$-module $\mathcal{F}_{e, \mathbf{s}_{l}}$, we need some combinatorial definitions.

Let $\underline{\lambda}=\left(\lambda^{(1)}, \ldots, \lambda^{(l)}\right)$ be a $l$-partition of rank $n$. The diagram of $\underline{\lambda}$ is the following set:

$$
[\underline{\lambda}]=\left\{(a, b, c) \mid 1 \leq c \leq l, 1 \leq b \leq \lambda_{a}^{(c)}\right\} .
$$

The elements of this diagram are called the nodes of $\underline{\lambda}$. Let $\gamma=(a, b, c)$ be a node of $\underline{\lambda}$. The residue of $\gamma$ associated to the set $\left\{e ; s_{1}, \ldots, s_{l}\right\}$ is the element of $\mathbb{Z} / e \mathbb{Z}$ defined by:

$$
\operatorname{res}(\gamma) \equiv\left(b-a+s_{c}\right)(\bmod e) .
$$

If $\gamma$ is a node with residue $i$, we say that $\gamma$ is an $i$-node. Let $\underline{\lambda}$ and $\mu$ be two $l$-partitions of rank $n$ and $n+1$ such that $[\underline{\lambda}] \subset[\mu]$. There exists a node $\gamma$ such that $[\mu]=[\underline{\lambda}] \cup\{\gamma\}$. Then, we denote $[\mu] /[\underline{\lambda}]=\gamma$ and if $\operatorname{res}(\gamma)=i$, we say that $\gamma$ is an addable $i$-node for $\underline{\lambda}$ and a removable $i$-node for $\mu$. Now, we introduce an order on the set of nodes of a $l$-partition. We say that $\gamma=(a, b, c)$ is above $\gamma^{\prime}=\left(a^{\prime}, b^{\prime}, c^{\prime}\right)$ if:

$$
b-a+s_{c}<b^{\prime}-a^{\prime}+s_{c^{\prime}} \text { or if } b-a+s_{c}=b^{\prime}-a^{\prime}+s_{c^{\prime}} \text { and } c^{\prime}<c .
$$

Let $\underline{\lambda}$ and $\mu$ be two $l$-partitions of rank $n$ and $n+1$ such that there exists an $i$-node $\gamma$ such that $[\underline{\mu}]=[\underline{\lambda}] \cup\{\gamma\}$. We define the following numbers:

$$
\begin{aligned}
N_{i}^{a}(\underline{\lambda}, \underline{\mu})= & \sharp\{\text { addable } i-\text { nodes of } \underline{\lambda} \text { above } \gamma\} \\
& -\sharp \text { removable } i \text {-nodes of } \underline{\mu} \text { above } \gamma\},
\end{aligned}
$$




$$
\begin{aligned}
N_{i}^{b}(\underline{\lambda}, \underline{\mu})= & \sharp\{\text { addable } i-\text { nodes of } \underline{\lambda} \text { below } \gamma\} \\
& -\sharp\{\text { removable } i-\text { nodes of } \underline{\mu} \text { below } \gamma\}, \\
N_{i}(\underline{\lambda})= & \sharp\{\text { addable } i-\text { nodes of } \underline{\lambda}\} \\
& -\sharp\{\text { removable } i-\text { nodes of } \underline{\lambda}\} .
\end{aligned}
$$

Theorem 3.1 $\mathcal{F}_{e, \mathbf{s}_{l}}$ is an integrable $\mathcal{U}_{q}^{\prime}\left(\widehat{\mathfrak{s l}_{e}}\right)$-module with action:

$$
\begin{aligned}
e_{i}\left|\underline{\lambda}, \mathbf{s}_{l}\right\rangle & =\sum_{\operatorname{res}([\underline{\underline{\lambda}}] /[\underline{\mu}]) \equiv i} q^{-N_{i}^{a}(\underline{\mu}, \underline{\lambda})}\left|\underline{\mu}, \mathbf{s}_{l}\right\rangle, \\
f_{i}\left|\underline{\lambda}, \mathbf{s}_{l}\right\rangle & =\sum_{\operatorname{res}([\underline{\mu}] /[\underline{\lambda}]) \equiv i} q^{N_{i}^{b}(\underline{\lambda}, \underline{\mu})}\left|\underline{\mu}, \mathbf{s}_{l}\right\rangle, \\
k_{i}\left|\underline{\lambda}, \mathbf{s}_{l}\right\rangle & =q^{N_{i}(\underline{\lambda})}\left|\underline{\lambda}, \mathbf{s}_{l}\right\rangle .
\end{aligned}
$$

where $0 \leq i \leq e-1$.

Note that this action coincides with that of [U] up to transformation $\left(s_{1}, \ldots, s_{l}\right) \mapsto$ $\left(s_{l}, \ldots, s_{1}\right)$.

We now introduce another basis for $\mathcal{F}_{e, \mathbf{s}_{l}}$ namely the Kashiwara-Lusztig canonical basis. This basis is defined by using an involution on the wedge space $\bigwedge^{s+\frac{\infty}{2}} V_{e, l}$ which has been introduced in [LT] for $l=1$ and generalized to any $l$ in [U]. Let $u_{\underline{k}}:=u_{k_{1}} \wedge u_{k_{2}} \wedge \ldots$ be a semi infinite monomial (ordered or not). For $i=1,2, \ldots$, we put $k_{i}=a_{i}+e\left(l-b_{i}\right)-e l m_{i}$ where $a_{i} \in\{1, \ldots, e\}, b_{i} \in\{1, \ldots, l\}$ and $m_{i} \in \mathbb{Z}$. For $r \in \mathbb{N}$, we put:

$$
\begin{aligned}
& \omega\left(u_{\underline{k}}\right)=\sharp\left\{i<j \mid a_{i}=a_{j}\right\}, \\
& \omega^{\prime}\left(u_{\underline{k}}\right)=\sharp\left\{i<j \mid b_{i}=b_{j}\right\} .
\end{aligned}
$$

Then, for $r \geq \sum_{i=1}^{\infty} k_{i}-(s-i+1)$ we set:

$$
\overline{u_{\underline{k}}}:=(-q)^{\omega^{\prime}\left(u_{\underline{k}}\right)} q^{-\omega\left(u_{\underline{k}}\right)} u_{k_{r}} \wedge u_{k_{r-1}} \wedge \ldots \wedge u_{k_{1}} \wedge u_{k_{r+1}} \wedge u_{k_{r+2}} \wedge \ldots
$$

One can prove that this monomial is independant of $r$ and that $u \mapsto \bar{u}$ defines an involution on $\bigwedge^{s+\frac{\infty}{2}} V_{e, l}$. The canonical basis is now defined as follows:

Theorem 3.2 Let $s \in \mathbb{Z}$. There exists a unique basis:

$$
\left\{G\left(\underline{\lambda}, \mathbf{s}_{l}\right) \mid \sum_{i=1}^{l} s_{i}=s, \underline{\lambda} \in \Pi_{l}^{n}, n \in \mathbb{N}\right\}
$$

of $\bigwedge^{s+\frac{\infty}{2}} V_{e, l}$ such that:

- $\overline{G\left(\underline{\lambda}, \mathbf{s}_{l}\right)}=G\left(\underline{\lambda}, \mathbf{s}_{l}\right)$,

- $G\left(\underline{\lambda}, \mathbf{s}_{l}\right)-\left|\underline{\lambda}, \mathbf{s}_{l}\right\rangle \in \bigoplus_{\underline{\mu}} q \mathbb{C}[q]\left|\underline{\mu}, \mathbf{s}_{l}\right\rangle$.

This is called the canonical basis of $\bigwedge^{s+\frac{\infty}{2}} V_{e, l}$. 
In particular the set $\left\{G\left(\underline{\lambda}, \mathbf{s}_{l}\right) \mid \underline{\lambda} \in \Pi_{l}^{n}, n \in \mathbb{N}\right\}$ gives a basis of the Fock space $\mathcal{F}_{e, \mathbf{s}_{l}}$. Now, we consider the subspace $M_{\mathbf{s}_{l}}:=\mathcal{U}_{q}^{\prime}\left(\widehat{\mathfrak{s l}_{e}}\right) \cdot\left|\underline{\emptyset}, \mathbf{s}_{l}\right\rangle$. It is well known that this is isomorphic to the irreducible $\mathcal{U}_{q}^{\prime}\left(\widehat{\mathfrak{s l}_{e}}\right)$-module $V(\Lambda)$ with highest weight $\Lambda:=\Lambda_{s_{1}(\bmod e)}+\Lambda_{s_{2}(\bmod e)}+\ldots+\Lambda_{s_{l}(\bmod e)}$. Note that if $\mathbf{s}_{l}^{\prime}=\left(s_{1}^{\prime}, \ldots, s_{l}^{\prime}\right) \in \mathbb{Z}^{l}$ is such that $s_{i} \equiv s_{i}^{\prime}(\bmod e)$, then the modules $M_{\mathbf{s}_{l}}$ and $M_{\mathbf{s}_{l}^{\prime}}$ are isomorphic (but the action of $\mathcal{U}_{q}^{\prime}\left(\widehat{\mathfrak{s t}_{e}}\right)$ on the elements of the standard basis $\left|\underline{\lambda}, \mathbf{s}_{l}\right\rangle$ and $\left|\underline{\lambda}, \mathbf{s}_{l}^{\prime}\right\rangle$ are different in general).

A basis of $M_{\mathbf{s}_{l}}$ can be given by using the canonical basis of $\mathcal{F}_{e, \mathbf{s}_{l}}$ and by studying the associated crystal graph. This graph can be described combinatorially as follows.

Let $\underline{\lambda}$ be a $l$-partition and let $\gamma$ be an $i$-node of $\underline{\lambda}$, we say that $\gamma$ is a normal $i$-node of $\underline{\lambda}$ if, whenever $\eta$ is an $i$-node of $\underline{\lambda}$ below $\gamma$, there are more removable $i$-nodes between $\eta$ and $\gamma$ than addable $i$-nodes between $\eta$ and $\gamma$. If $\gamma$ is the highest normal $i$-node of $\underline{\lambda}$, we say that $\gamma$ is a good $i$-node. Note that this notion depends on the choice of $\mathbf{s}_{l}$.

Then, the crystal graph of $\mathcal{F}_{e, \mathbf{s}_{l}}$ is given by:

- vertices: the $l$-partitions,

- edges: $\underline{\lambda} \stackrel{i}{\rightarrow} \underline{\mu}$ if and only if $[\underline{\mu}] /[\underline{\lambda}]$ is a good $i$-node.

By using properties of crystal bases, we can obtain the crystal graph of $M_{\mathbf{s}_{l}}$ : this is the connected composants of that of $\mathcal{F}_{e, \mathbf{s}_{l}}$ which contain the vacuum vector $\left|\underline{\emptyset}, \mathbf{s}_{l}\right\rangle$. The vertices of this graph are given by the following class of $l$-partitions.

Definition 3.3 Let $\mathbf{s}_{l} \in \mathbb{Z}^{n}$. The set of Uglov l-partitions $\Lambda_{e ; \mathbf{s}_{l}}^{n}$ is defined recursively as follows.

- We have $\underline{\emptyset}:=(\emptyset, \emptyset, \ldots, \emptyset) \in \Lambda_{e ; \mathbf{s}_{l}}^{n}$.

- If $\underline{\lambda} \in \Lambda_{e ; \mathbf{s}_{l}}^{n}$, there exist $i \in\{0, \ldots, e-1\}$ and a good $i$-node $\gamma$ such that if we remove $\gamma$ from $\underline{\lambda}$, the resulting l-partition is in $\Lambda_{e ; \mathbf{s}_{l}}^{n}$.

Remark 3.4 Assume that $\mathbf{s}_{l} \in \mathbb{Z}^{n}$ is such that $0 \leq s_{1} \leq s_{2} \leq \ldots \leq s_{l}<e$ then it is shown in [FL] that the set of Uglov l-partitions are the l-partitions $\underline{\lambda}=\left(\lambda^{(1)}, \ldots, \lambda^{(l)}\right)$ such that:

1. for all $1 \leq j \leq l-1$ and $i=1,2, \ldots$, we have:

$$
\begin{aligned}
& \lambda_{i}^{(j+1)} \geq \lambda_{i+s_{j+1}-s_{j}}^{(j)} \\
& \lambda_{i}^{(1)} \geq \lambda_{i+e+s_{1}-s_{l}}^{(l)}
\end{aligned}
$$

2. for all $k>0$, among the residues appearing at the right ends of the length $k$ rows of $\underline{\lambda}$, at least one element of $\{0,1, \ldots, e-1\}$ does not occur.

Such l-partitions are called FLOTW l-partitions in $[J p \mid$.

Assume that $\mathbf{s}_{l} \in \mathbb{Z}^{n}$ is such that $s_{1}<<s_{2}<<\ldots<<s_{l}$, then the set Uglov l-partitions $\Lambda_{e ; \mathbf{s}_{l}}^{n}$ coincides with the set of Kleshchev l-partitions as in [Ac]. 
Now, the canonical basis of $M_{\mathbf{s}_{l}}$ is the following set:

$$
\left\{G\left(\underline{\lambda}, \mathbf{s}_{l}\right) \mid \underline{\lambda} \in \Lambda_{e ; \mathbf{s}_{l}}^{n}, n \in \mathbb{N}\right\} .
$$

The following theorem gives a link between the canonical basis elements of $M_{\mathbf{s}_{l}}$ and the decomposition matrices of Ariki-Koike algebras.

Theorem 3.5 (Ariki, see [Ab]) Let $\mathcal{H}_{\mathbb{C}, n}$ be the Ariki-Koike algebra over $\mathbb{C}$ with the following choice of parameters:

$$
\begin{aligned}
& x_{j}=\eta_{e}^{v_{j}} \text { for } j=1, \ldots, l, \\
& v=\eta_{e},
\end{aligned}
$$

where $0 \leq v_{1} \leq \ldots \leq v_{l}<e$. Let $\Phi^{n}:=\Phi_{\left\{e ; v_{l}, \ldots, v_{l}\right\}}^{n}$ and $\left(d_{\underline{\lambda}, \underline{\mu}}\right)_{\underline{\lambda} \in \Pi_{l}^{n}, \underline{\mu} \in \Phi^{n}}$ be the associated decomposition matrix.

Let $\mathbf{s}_{l}=\left(s_{1}, \ldots, s_{l}\right) \in \mathbb{Z}^{l}$ be such that $v_{j} \equiv s_{j}(\bmod e)$ for $j=1, \ldots, l$. Then, for each $\underline{\nu} \in \Lambda_{e ; \mathbf{s}_{l}}^{n}$, write

$$
G\left(\underline{\nu}, \mathbf{s}_{l}\right)=\sum_{\underline{\lambda} \in \Pi_{l}^{n}} d_{\underline{\lambda}, \underline{\nu}}(q)\left|\underline{\lambda}, \mathbf{s}_{l}\right\rangle
$$

where $d_{\underline{\lambda}, \underline{\nu}}(q) \in \mathbb{C}[q]$. Then there exists a bijection $\kappa: \Phi^{n} \rightarrow \Lambda_{e ; \mathbf{s}_{l}}^{n}$ such that for all $\underline{\lambda} \in \Pi_{l}^{n}$ and $\underline{\mu} \in \Phi^{n}$, we have:

$$
d_{\underline{\lambda}, \underline{\mu}}=d_{\underline{\lambda}, \kappa(\underline{\mu})}(1) .
$$

In other words, Ariki's theorem asserts that the columns of the decomposition matrix of $\mathcal{H}_{\mathbb{C}, n}$ with the above choice of parameters coincides with the canonical basis elements of $M_{\mathbf{s}_{l}}$ evaluated at $q=1$ whenever $v_{j} \equiv s_{j}(\bmod e)$ for $j=1, \ldots, l$. Note that this decomposition matrix only depends on $\left\{e ; v_{1}, \ldots, v_{l}\right\}$ whereas the canonical basis elements of $M_{\mathbf{s}_{l}}$ depends on $\left\{e ; s_{1}, \ldots, s_{l}\right\}$. In particular, if $s_{j} \equiv s_{j}^{\prime}(\bmod e)$ for $j=1, \ldots, l$, the labelings of the canonical basis elements of $M_{\mathbf{s}_{l}}$ and $M_{\mathbf{s}_{l}^{\prime}}$ by $\Lambda_{e ; \mathbf{s}_{l}}^{n}$ and $\Lambda_{e ; \mathbf{s}_{l}^{\prime}}^{n}$ are different in general as we can see in the following example.

Example 3.6 Assume that $l=2, e=4, v_{1}=0, v_{2}=1$. Then different values for $\mathbf{s}_{l}$ lead to different labelings of the same crystal graph:

- for $s_{1}=0 \equiv v_{1}(\bmod e), s_{2}=1 \equiv v_{2}(\bmod e)$ :

$$
\Lambda_{\mathbf{s}_{l}}^{4}=\{(\emptyset,(4)) ;((1),(2,1)) ;((1,1),(1,1)) ;((1),(3)) ;((1,1),(2)) ;((2),(1,1)) ;
$$$$
((2),(2)) ;((2,1),(1)) ;((2,1,1), \emptyset) ;((2,2), \emptyset) ;((3),(1)) ;((3,1), \emptyset) ;((4), \emptyset)\} \text {; }
$$

- for $s_{1}=4 \equiv v_{1}(\bmod e), s_{2}=1 \equiv v_{2}(\bmod e)$ :

$$
\begin{gathered}
\Lambda_{\mathbf{s}_{l}}^{4}=\{((1,1,1),(1)) ;((1),(2,1) ;((1,1),(1,1)) ;((1),(3)) ;((1,1),(2)) ; \\
((2),(1,1)) ;((2),(2)) ;((2,1),(1)) ;((2,1,1), \emptyset) ;((2,2), \emptyset) ;((3),(1)) ; \\
((3,1), \emptyset) ;((4), \emptyset)\} ;
\end{gathered}
$$


- for $s_{1}=0 \equiv v_{1}(\bmod e), s_{2}=5 \equiv v_{2}(\bmod e)$ :

$$
\begin{gathered}
\Lambda_{\mathbf{s}_{l}}^{4}=\{(\emptyset,(2,1,1)) ;(\emptyset,(2,2)),((1),(1,1,1)) ;((1,1),(1,1)) ;((1,1),(2)) ; \\
((2),(1,1)) ;((1),(2,1)) ;((1,1,1),(1)) ;((1,1),(2)) ;((1),(3)) ;(\emptyset,(3,1)) ; \\
(\emptyset,(4)) ;((2,1),(1)),((2),(2))\} .
\end{gathered}
$$

In $\left[\mathrm{Ac}\right.$, Ariki has given an explicit description of $\Phi_{\left\{e ; v_{1}, \ldots, v_{l}\right\}}^{n}$ by showing that this set coincides with the set $\Lambda_{e ; \mathbf{s}_{l}}^{n}$ with $s_{1}>>s_{2}>>\ldots>>s_{l}$ and $v_{j} \equiv s_{j}(\bmod e)$ for $j=1, \ldots, l$. In $[\mathrm{Jp}$, another parametrization of the simple $\mathcal{H}_{\mathbb{C}, n}$-modules has been given by using the set $\Lambda_{e ; \mathbf{s}_{l}}^{n}$ with $\mathbf{s}_{l}=\left(v_{1}, \ldots, v_{l}\right)$ (namely the set of FLOTW $l$-partitions) and an ordering of the rows of the decomposition matrices by Lusztig $a$-values. In the next section, we will show that each of the sets $\Lambda_{e ; \mathbf{s}_{l}}^{n}$ with $v_{j} \equiv s_{j}(\bmod e)$ has a natural interpretation in the representation theory of $\mathcal{H}_{\mathbb{C}, n}$.

\section{Unitriangularity of the decomposition matri- ces of Ariki-Koike algebras}

\section{A Specialisations and Lusztig $a$-values}

Let $e$ be an integer and let $\mathbf{s}_{l}=\left(s_{1}, s_{2}, \ldots, s_{l}\right) \in \mathbb{Z}^{l}$ be a sequence of integers. The aim of this part is to study the Ariki-Koike algebra $\mathcal{H}_{\mathbb{C}, n}$ with the following choice of parameters:

$$
\begin{aligned}
& u_{j}=\eta_{e}^{s_{j}(\bmod e)} \text { for } j=1, \ldots, l, \\
& v=\eta_{e} .
\end{aligned}
$$

For $j=1, \ldots, l$, we define rational numbers:

$$
m^{(j)}=s_{j}-\frac{(j-1) e}{l}+\alpha e,
$$

where $\alpha$ is a positive integer such that $m^{(j)} \geq 0$ for $j=1, \ldots, l$. Let $y$ be an indeterminate and put $A:=\mathbb{C}\left[y, y^{-1}\right]$. We consider the Ariki-Koike algebra $\mathcal{H}_{A, n}^{\mathbf{s}_{l}}$ over $A$ with the following parameters:

$$
\begin{aligned}
& u_{j}=y^{l m^{(j)}} \eta_{l}^{j-1} \text { for } j=1, \ldots, l, \\
& v=y^{l} .
\end{aligned}
$$

By Theorem 2.2] $\mathcal{H}_{\mathbb{C}(y), n}^{\mathbf{s}_{l}}:=\mathbb{C}(y) \otimes_{A} \mathcal{H}_{A, n}^{\mathbf{s}_{l}}$ is split semisimple. Moreover, if we specialize the parameter $y$ to $\eta_{l e}:=\exp \left(\frac{2 i \pi}{l e}\right)$, we obtain the above ArikiKoike algebra $\mathcal{H}_{\mathbb{C}, n}$. Hence, we have a well-defined decomposition map between $R_{0}\left(\mathcal{H}_{\mathbb{C}(y), n}^{\mathbf{s}_{l}}\right)$ (which can be identified with $\mathcal{F}_{n}$ as it is explained in section 2) and $R_{0}\left(\mathcal{H}_{\mathbb{C}, n}\right)$.

We now associate to each simple $\mathcal{H}_{\mathbb{C}(y), n}^{\mathbf{s}_{l}}$-module $S_{\mathbb{C}(y)}^{\lambda}$ an $a$-value following Jp. Put $\mathbf{m}_{l}=\left(m^{(1)}, \ldots, m^{(l)}\right)$ where the $m^{(j)}$ are defined above. We need to define the notion of " $\mathbf{m}_{l}$-translated symbols" associated to $l$-compositions.

Let $n \in \mathbb{N}$ and $l \in \mathbb{N}$. An $l$-composition $\underline{\lambda}$ of rank $n$ is an $l$-tuple $\left(\lambda^{(1)}, \ldots ., \lambda^{(l)}\right)$ where : 
- for all $i=1, \ldots, l$, we have $\lambda^{(i)}=\left(\lambda_{1}^{(i)}, \ldots, \lambda_{h^{(i)}}^{(i)}\right)$ for $h^{(i)} \in \mathbb{N}$ and $\lambda_{j}^{(i)} \in \mathbb{N}_{>0}$ $\left(j=1, \ldots, h^{(i)}\right), h^{(i)}$ is called the height of $\lambda^{(i)}$,

- $\sum_{i=1}^{l} \sum_{j=1}^{h^{(i)}} \lambda_{j}^{(i)}=n$.

Let $\underline{\lambda}=\left(\lambda^{(1)}, \ldots, \lambda^{(l)}\right)$ be a $l$-composition and let $h^{(i)}$ be the heights of the compositions $\lambda^{(i)}$. Then the height of $\underline{\lambda}$ is the following positive integer:

$$
h_{\underline{\lambda}}:=\max \left\{h^{(1)}, \ldots, h^{(l)}\right\} .
$$

Let $k$ be a positive integer. The translated symbol $\mathbf{B}\left[\mathbf{m}_{l}\right]^{\prime}$ associated to $\mathbf{m}_{l}, k$ and $\underline{\lambda}$ is:

$$
\mathbf{B}\left[\mathbf{m}_{l}\right]^{\prime}:=\left(B^{\prime(1)}, \ldots, B^{\prime(l)}\right),
$$

where $B^{\prime(i)}$, for $i=1, \ldots, l$, is given by:

$$
B^{\prime(i)}:=\left(B_{1}^{\prime(i)}, \ldots, B_{h_{\underline{\lambda}}+k}^{(i)}\right)
$$

in which:

$$
B_{j}^{(i)}:=\lambda_{j}^{(i)}-j+h_{\underline{\lambda}}+k+m^{(i)} \quad\left(1 \leq j \leq h_{\underline{\lambda}}+k\right) .
$$

The integer $h_{\underline{\lambda}}+k$ is called the height of $\mathbf{B}\left[\mathbf{m}_{l}\right]^{\prime}$.

Now, the $a$-value on the $\mathcal{H}_{\mathbb{C}(y), n}^{\mathbf{s}_{l}}$-module $S \frac{\lambda}{\mathbb{C}(y)}$ is defined by using the characterization of the Schur elements which has been obtained by Geck, Iancu and Malle in GIM]. We obtain the following definition:

Definition 4.1 Let $\underline{\lambda}$ be a l-partition of rank $n$ and let $S_{\mathbb{C}(y)}$ be the simple $\mathcal{H}_{\mathbb{C}(y), n}^{\mathbf{s}_{l}}$-module. Let $k$ be a positive integer and $\boldsymbol{B}\left[\mathbf{m}_{l}\right]^{\prime}$ be the set associated to $m$ and $k$. Then, if $h$ is the height of $\boldsymbol{B}\left[\mathbf{m}_{l}\right]^{\prime}$, the a-value of $S \frac{\lambda}{\mathbb{C}(y)}$ is the following rational number:

$$
a_{\mathbf{s}_{l}}(\underline{\lambda})=f\left(n, h, \mathbf{m}_{l}\right)+\sum_{\substack{1 \leq i \leq j<l+1 \\(a, b) \in B^{\prime}(i) \times B^{\prime}(j) \\ a>b} \text { if } i=j} \min \{a, b\}-\sum_{\substack{1 \leq i, j<l+1 \\ a \in B^{\prime}(i) \\ 1 \leq k \leq a}} \min \left\{k, m^{(j)}\right\},
$$

where $f\left(n, h, \mathbf{m}_{l}\right)$ is a rational number which only depends on the parameters $\left\{e ; s_{1}, \ldots, s_{l}\right\}$, on $h$ and on $n$ (the expression of $f$ is given in $\left.[J p]\right)$.

We need to introduce the following preorder on the set of $l$-compositions.

Definition 4.2 Let $\mu$ and $\underline{\nu}$ be l-compositions of rank $n$. Let $k$ and $k^{\prime}$ be such that $h_{\underline{\mu}}+k=h_{\underline{\nu}}+k^{\prime}$. Let $\bar{B}_{\underline{\mu}}\left[\mathbf{m}_{l}\right]^{\prime}$, (resp. $\boldsymbol{B}_{\underline{\underline{\nu}}}\left[\mathbf{m}_{l}\right]^{\prime}$ ) be the $m$-translated symbol associated to $\underline{\mu}, m$ and $k$ (resp. $\underline{\mu}, \mathbf{m}_{l}$ and $k^{\prime}$ ). Then we write:

$$
\underline{\mu} \prec \underline{\nu},
$$

if:

$$
\sum_{\substack{1 \leq i \leq j<l+1 \\(a, b) \in B_{\underline{\underline{\mu}}}^{\prime(i)} \times B_{\underline{\mu}}^{\prime(j)} \\ a>b^{\text {if }} i=j}} \min \{a, b\}-\sum_{\substack{1 \leq i, j<l+1 \\ a \in B_{\mu}^{\prime(i)} \\ 1 \leq k \leq a}} \min \left\{k, m^{(j)}\right\}<
$$




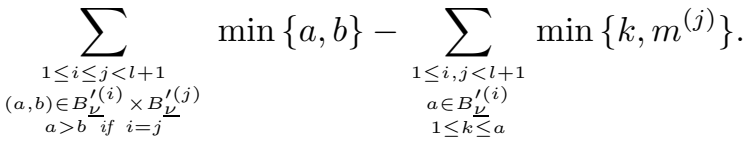

In particular, if $\underline{\mu}$ and $\underline{\nu}$ are l-partitions, we have:

$$
\underline{\mu} \prec \underline{\nu} \Longleftrightarrow a_{\mathbf{s}_{l}}(\underline{\mu})<a_{\mathbf{s}_{l}}(\underline{\nu}) .
$$

Before beginning the proof of the main result, we need to give the following useful proposition which has been shown in Jp in the case $0 \leq s_{1} \leq \ldots \leq s_{l}<e$. One can easily check that the proof holds in the general case.

Proposition 4.3 Let $\underline{\lambda}$ be a l-composition of rank $n$, let $\boldsymbol{B}:=\left(B^{(1)}, \ldots, B^{(l)}\right)$ be an ordinary symbol of $\underline{\lambda}$, let $\beta_{1}$ and $\beta_{2}$ be two elements of $\boldsymbol{B}\left[\mathbf{m}_{l}\right]^{\prime}$, we assume that:

$$
\beta_{1}<\beta_{2} .
$$

Let $r \in \mathbb{N}$. We add $r$ nodes to $\underline{\lambda}$ on the part associated to $\beta_{1}$. Let $\underline{\mu}$ be the resulting l-composition of rank $n+r$. We add $r$ nodes to $\underline{\lambda}$ on the part associated to $\beta_{2}$. Let $\underline{\nu}$ be the resulting $l$-composition of rank $n+r$. Then, we have:

$$
\underline{\nu} \prec \underline{\mu} .
$$

In particular, if $\underline{\mu}$ and $\underline{\nu}$ are some l-partitions, then:

$$
a_{\mathbf{s}_{l}}(\underline{\mu})>a_{\mathbf{s}_{l}}(\underline{\nu}) .
$$

Assume that $\underline{\lambda}$ is a l-composition of rank $n$.

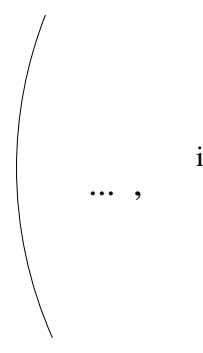

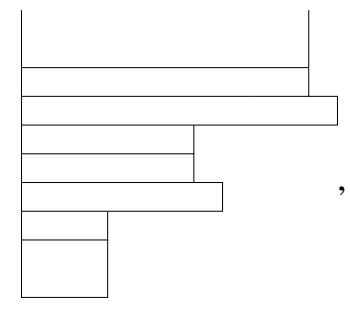

$\mathrm{N}$
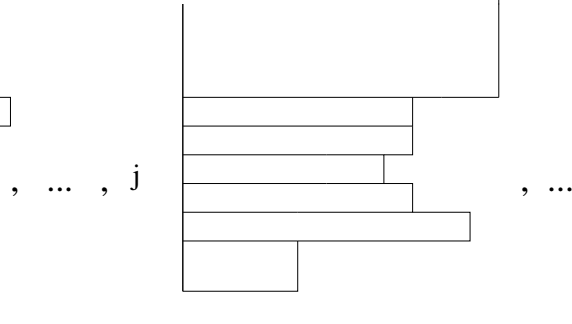

$N^{\prime}$

Assume that $\underline{\mu}$ and $\underline{\mu}^{\prime}$ are two l-compositions which are obtained from $\underline{\lambda}$ by adding $r$ nodes on the parts $\lambda_{i}^{(N)}$ and $\lambda_{j}^{\left(N^{\prime}\right)}$ respectively. Assume in addition that we have:

$$
\lambda_{i}^{(N)}-i+s_{N}-\frac{N e}{l}>\lambda_{j}^{\left(N^{\prime}\right)}-j+s_{N^{\prime}}-\frac{N^{\prime} e}{l} .
$$

Observe that if we use the notation of Section $3 . \mathrm{A}$ this property is equivalent to $k_{i}^{(N)}-\frac{N e}{l}>k_{j}^{\left(N^{\prime}\right)}-\frac{N^{\prime} e}{l}$.

Keeping the above representation of $\underline{\lambda}, \underline{\mu}$ and $\underline{\mu}^{\prime}$ are respectively given as follows: 


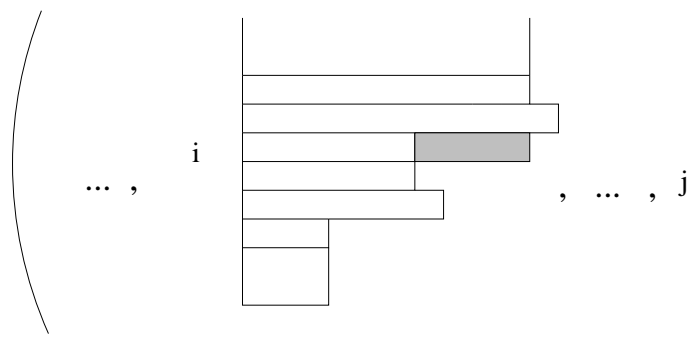

$\mathrm{N}$
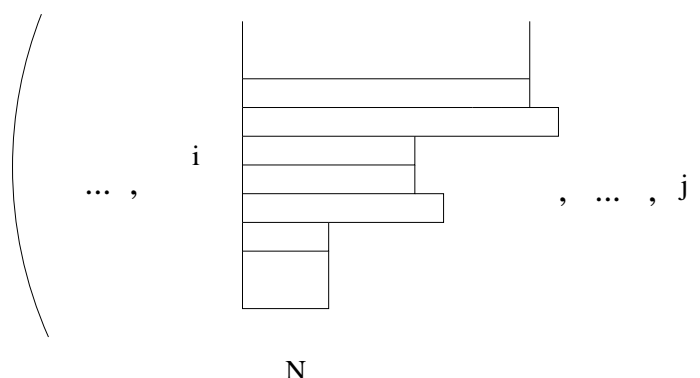

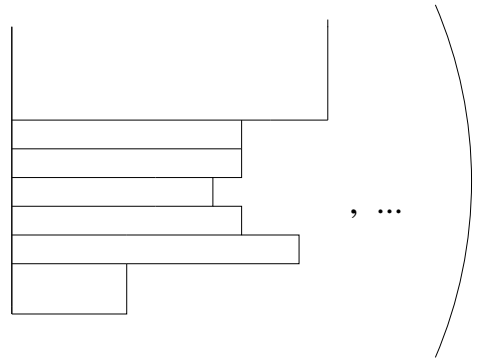

N'

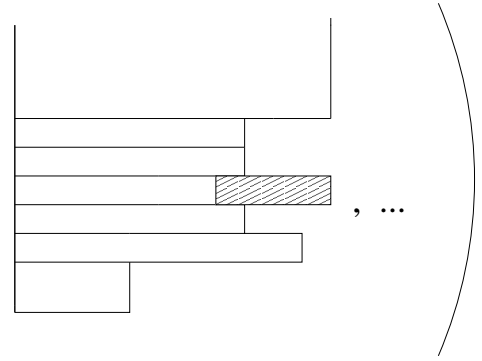

N'

Then Proposition 4.3 asserts that we have:

$$
\underline{\mu} \prec \underline{\mu^{\prime}} .
$$

The aim of the following part is to show that the matrix associated to the canonical basis elements of the irreducible modules $M_{\mathbf{s}_{l}}$ is unitriangular with respect to $a$-values.

\section{B Study of the involution on the semi-infinite $q$-wedge product}

Let $s \in \mathbb{Z}$. We keep the notations of the previous sections. We will work with the semi-infinite $q$-wedge product $\bigwedge^{s+\frac{\infty}{2}} V_{e, l}$. First, we attach to each semiinfinite monomial an $a$-value. Let $u_{\underline{k}} \in \bigwedge^{s+\frac{\infty}{2}} V_{e, l}$ be a semi-infinite $q$-wedge product and let $u_{\tilde{k}}$ be the monomial obtained by reordering the $k_{i}$ in strictly decreasing order. Then, the bijection described in section 3.A shows that we can associate to $u_{\underline{\tilde{k}}}$ a symbol $\left|\underline{\lambda}, \mathbf{s}_{l}\right\rangle$ with $\underline{\lambda} \in \Pi_{l}^{n}$ and $\mathbf{s}_{l}=\left(s_{1}, \ldots, s_{l}\right) \in \mathbb{Z}^{l}$ verifying $\sum_{i=1}^{l} s_{i}=s$. We put:

$$
\begin{aligned}
& \pi\left(u_{\underline{k}}\right):=\left|\underline{\lambda}, \mathbf{s}_{l}\right\rangle, \\
& a\left(u_{\underline{k}}\right):=a_{\mathbf{s}_{l}}(\underline{\lambda}) .
\end{aligned}
$$

In this part, we show the following proposition:

Proposition 4.4 Let $u_{\underline{k}} \in \bigwedge^{s+\frac{\infty}{2}} V_{e, l}$ be a semi-infinite ordered q-wedge product. Then, we have:

$$
\overline{u_{\underline{k}}}=u_{\underline{k}}+\text { sum of ordered monomials } u_{\underline{r}} \text { with } a\left(u_{\underline{r}}\right)>a\left(u_{\underline{k}}\right),
$$

where the involution is defined in section 3.B. 
We note that a similar property is shown in [U] but for a (partial) order which is not compatible with the preorder induced by the $a$-values.

Let $u_{\underline{k}}$ be an arbitrary semi-infinite monomial such that there exists $i \in \mathbb{N}$ such that $k_{i}<k_{i+1}$. Then the relations $(R 1)-(R 4)$ in section 3.A] show how to express $u_{\underline{k}}$ in terms of semi-infinite monomials $u_{\underline{k}^{\prime}}$ such that $k_{i}^{\prime}>k_{i+1}^{\prime}$. Let $u_{\underline{k^{\prime}}}$ be such a semi-infinite monomial such that $k_{i}^{\prime} \neq k_{i+1}$ and $k_{i+1}^{\prime} \neq k_{i}$. Then the $l$-partitions associated to $u_{\underline{k}}$ and $u_{\underline{k}^{\prime}}$ have the same charge $\mathbf{s}_{l}$. We put :

$$
\begin{aligned}
\left|\underline{\lambda}, \mathbf{s}_{l}\right\rangle & :=\pi\left(u_{\underline{k}}\right), \\
\left|\underline{\lambda^{\prime}}, \mathbf{s}_{l}\right\rangle & :=\pi\left(u_{\underline{k^{\prime}}}\right) .
\end{aligned}
$$

From the relations $(R 1)-(R 4)$, using the representations of $u_{\underline{k}}$ and $u_{\underline{k^{\prime}}}$ by $l$ abacus, we can see that $u_{\underline{k^{\prime}}}$ is obtained from $u_{\underline{k}}$ by moving two colored beads representing $k_{1}$ and $k_{2}$ to two colored beads representing $k_{1}^{\prime}$ and $k_{2}^{\prime}$ and lying in the same runners as $k_{1}$ and $k_{2}$. As a consequence, the $l$-partition $\underline{\lambda}^{\prime}$ is obtained from $\underline{\lambda}$ by removing a ribbon $R$ of size $x$ from a component $N$ and adding a ribbon $R^{\prime}$ of size $x$ to a component $N^{\prime}$ as in the following figure.

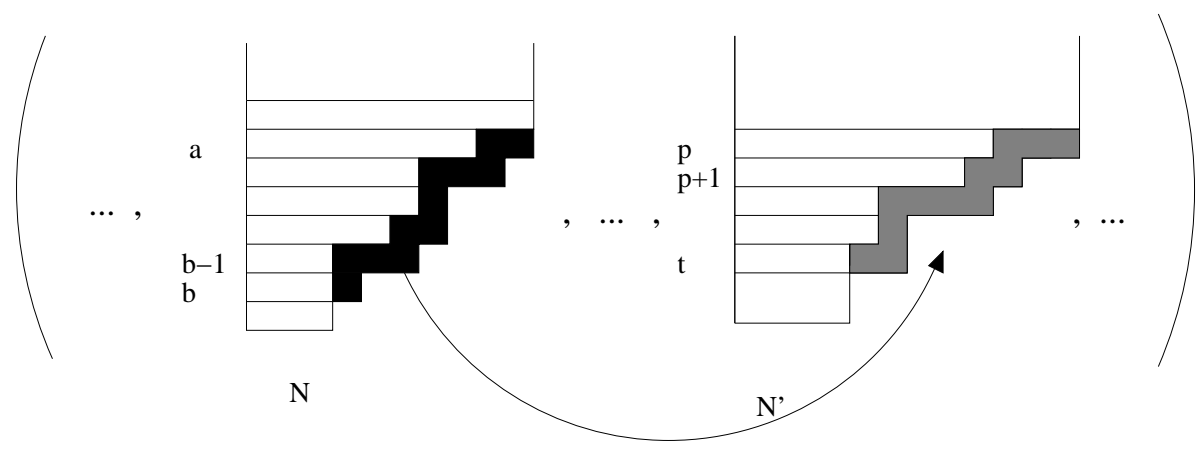

Then, there exist positive integers $a, b, p$ and $t$ such that:

$$
\begin{aligned}
& \lambda^{\prime(N)}=\left(\lambda_{1}^{(N)}, \ldots, \lambda_{a-1}^{(N)}, \lambda_{a+1}^{(N)}-1, \ldots, \lambda_{b}^{(N)}-1, \lambda_{a}^{(N)}-(x-(a+b)), \lambda_{b+1}^{(N)}, \ldots, \lambda_{h^{(N)}}^{(N)}\right), \\
& \lambda^{\prime\left(N^{\prime}\right)}=\left(\lambda_{1}^{\left(N^{\prime}\right)}, \ldots, \lambda_{p-1}^{\left(N^{\prime}\right)}, \lambda_{t}^{\left(N^{\prime}\right)}+(x-(p-k)), \lambda_{p}^{\left(N^{\prime}\right)}+1, \ldots, \lambda_{t-1}^{\left(N^{\prime}\right)}+1, \lambda_{t+1}^{\left(N^{\prime}\right)}, \ldots, \lambda_{h^{\left(N^{\prime}\right)}}^{\left(N^{\prime}\right)}\right) .
\end{aligned}
$$

In fact, the two $l$-partitions $\underline{\lambda}^{\prime}$ and $\underline{\lambda}$ are both obtained by adding $R$ and $R^{\prime}$ to the components $N$ and $N^{\prime}$ of the same $l$-partition $\underline{\nu}=\pi\left(u_{\underline{r}}\right)$. Here, $u_{\underline{r}}$ corresponds to the sequence $\left(k_{1}, \ldots, k_{i-1}, \hat{k}_{i}, \hat{k}_{i+1}, k_{i+2}, \ldots\right)$ where $\hat{k}_{i}$ and $\hat{k}_{i+1}$ are the minimal integers of $\left\{k_{i}, k_{i+1}, k_{i}^{\prime}, k_{i+1}^{\prime}\right\}$ such that the beads associated to $\hat{k}_{i}$ and $\hat{k}_{i+1}$ lie at the components $N$ and $N^{\prime}$ of the $l$-abacus of $u_{\underline{r}}$ and $\hat{k}_{i+1}<\hat{k}_{i}$.

Assume that $\underline{\lambda} \neq \underline{\lambda}^{\prime}$. Put $\hat{k}_{i}=a+e(l-b)-e l m$ and $\hat{k}_{i+1}=a^{\prime}+e\left(l-b^{\prime}\right)-e l m^{\prime}$ where $a, a^{\prime} \in\{1, \ldots, e\}, b, b^{\prime} \in\{1, \ldots, l\}$ and, $m, m^{\prime} \in \mathbb{Z}$. Then, by studying the relations $(R 1)-(R 4)$, we get that:

$$
a-e m-\frac{N e}{l}>a^{\prime}-e m^{\prime}-\frac{N^{\prime} e}{l} .
$$

Thus, we obtain:

$$
\nu_{b}^{(N)}-b+s_{N}-\frac{N e}{l}>\nu_{t}^{\left(N^{\prime}\right)}-t+s_{N^{\prime}}-\frac{N^{\prime} e}{l} .
$$


Assume that we have $\lambda_{i}^{\prime\left(N^{\prime}\right)}=\nu_{i}^{\left(N^{\prime}\right)}+y_{i}$ for integers $y_{i}$ with $i=p, p+1, \ldots, t$ and assume that we have $\lambda_{j}^{(N)}=\nu_{j}^{(N)}+x_{i}$ for integers $x_{j}$ with $j=a, a+1, \ldots b$. We define a $l$-composition $\underline{\lambda}[1]$ as follows.

- If $y_{t} \leq x_{b}$, then we put $j_{1}:=b$ and we define:

$$
\underline{\lambda}[1]_{i}^{(k)}= \begin{cases}\lambda_{i}^{\prime(k)} & \text { if }(i, k) \neq(b, N) \text { and }(i, k) \neq\left(t, N^{\prime}\right), \\ \lambda_{i}^{\prime(k)}+y_{t} & \text { if }(i, k)=(b, N), \\ \lambda_{i}^{\prime(k)}-y_{t} & \text { if }(i, k)=\left(t, N^{\prime}\right) .\end{cases}
$$

In this case, $\underline{\lambda}[1]$ and $\underline{\lambda}^{\prime}$ are both obtained by adding the same number of nodes on a $l$-composition. Then, by $(*)$ and by Proposition 4.3 , we get:

$$
\underline{\lambda}[1] \prec \underline{\lambda}^{\prime}
$$

- If $y_{t}>x_{b}$, there exists $j_{1} \in\{a, a+1, \ldots, b-1\}$ such that:

$$
x_{j_{1}}+x_{j_{1}+1}+\ldots+x_{b} \geq y_{t} \geq x_{j_{1}+1}+\ldots+x_{b-1}+x_{b} .
$$

Then, we define:

$$
\underline{\lambda}[1]_{i}^{(k)}= \begin{cases}\lambda_{i}^{\prime(k)}+x_{i} & \text { if } k=N \text { and } \\ \lambda_{i}^{\prime(k)}+y_{t}-\left(x_{j_{1}+1}+\ldots+x_{b-1}+x_{b}\right) & \text { if }(i, k)=\left(j_{1}, N\right), \\ \lambda_{i}^{\prime(k)}-y_{t} & \text { if }(i, k)=\left(t, N^{\prime}\right), \\ \lambda_{i}^{\prime(k)} & \text { if otherwise. }\end{cases}
$$

For all $i$ such that $a \leq i \leq b$, observe that $\nu_{b}^{(N)}+x_{i+1}+\ldots+x_{b-1}+x_{b}=$ $\nu_{i}+(b-i)$. Then, by $(*)$, for all $i$ such that $j_{1} \leq i \leq b$, we have:

$$
\nu_{i}^{(N)}-i+s_{N}-\frac{N e}{l}>\nu_{t}^{\left(N^{\prime}\right)}-t+x_{i+1}+\ldots+x_{b}+s_{N^{\prime}}-\frac{N^{\prime} e}{l} .
$$

Thus, for all $i$ such that $j_{1} \leq i \leq b$, we obtain:

${\lambda^{\prime}}_{i}^{(N)}-i+s_{N}-\frac{N e}{l}>{\lambda^{\prime}}_{t}^{\left(N^{\prime}\right)}-t+x_{i+1}+\ldots+x_{b-1}+x_{b}-y_{t}+s_{N^{\prime}}-\frac{N^{\prime} e}{l}$.

Thus, using Proposition 4.3 we get:

$$
\underline{\lambda}[1] \prec \underline{\lambda}^{\prime} .
$$

Keeping the above figure, $\underline{\lambda}[1]$ is as follows.

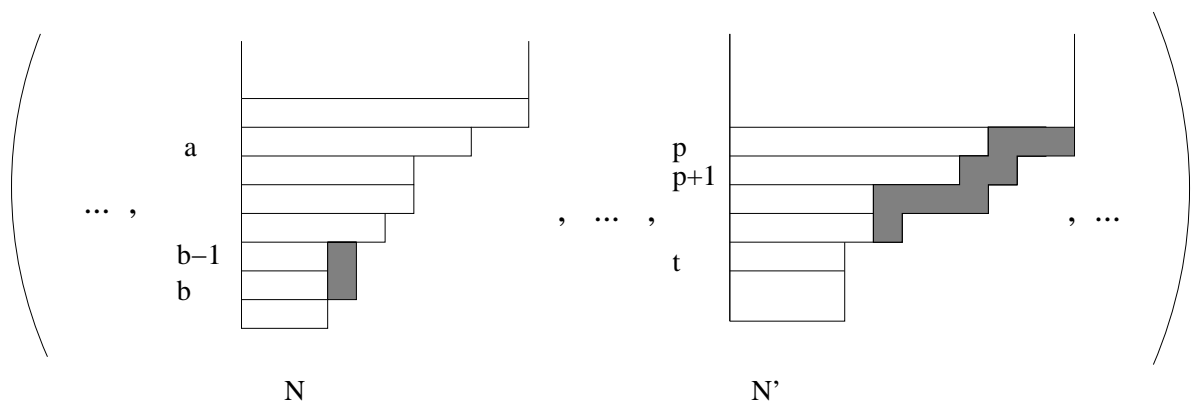


As we have $\nu_{t}^{\left(N^{\prime}\right)}-1+y_{t}=\nu_{t-1}^{\left(N^{\prime}\right)}$, note that:

$$
\lambda[1]_{j_{1}}^{(N)}-j_{1}+s_{N}-\frac{N e}{l}>\nu_{t-1}^{\left(N^{\prime}\right)}-(t-1)+s_{N^{\prime}}-\frac{N^{\prime} e}{l} .
$$

We now define a l-composition $\underline{\lambda}[2]$ as follows. Put:

$$
x_{j_{1}}^{\prime}:=x_{j_{1}}+x_{j_{1}+1}+\ldots+x_{b}-y_{t} .
$$

- If $y_{t-1} \leq x_{j_{1}}^{\prime}$, we put $j_{2}:=j_{1}$ and we define:

$$
\underline{\lambda}[2]_{i}^{(k)}= \begin{cases}\lambda[1]_{i}^{(k)} & \text { if }(i, k) \neq\left(j_{1}, N\right) \text { and }(i, k) \neq\left(t-1, N^{\prime}\right), \\ \lambda[1]_{i}^{(k)}+y_{t-1} & \text { if }(i, k)=\left(j_{1}, N\right), \\ \lambda[1]_{i}^{(k)}-y_{t-1} & \text { if }(i, k)=\left(t-1, N^{\prime}\right) .\end{cases}
$$

By $(* *)$, we have:

$$
\lambda[1]_{j_{1}}^{(N)}-j_{1}+s_{N}-\frac{N e}{l}>\lambda[1]_{t-1}^{\left(N^{\prime}\right)}-y_{t-1}-(t-1)+s_{N}-\frac{N^{\prime} e}{l}
$$

By Proposition 4.3 we get:

$$
\underline{\lambda}[2] \prec \underline{\lambda}[1] .
$$

- If $y_{t-1}>x_{j_{1}}^{\prime}$, there exists $j_{2} \in\left\{a, a+1, \ldots, j_{1}-1\right\}$ such that:

$$
x_{j_{2}}+x_{j_{2}+1}+\ldots+x_{j_{1}-1}+x_{j_{1}}^{\prime} \geq y_{t-1}>x_{j_{2}+1}+\ldots+x_{j_{1}-1}+x_{j_{1}}^{\prime} .
$$

Then, we define:

$$
\underline{\lambda}[2]_{i}^{(k)}= \begin{cases}\lambda[1]_{i}^{(k)}+x_{j_{1}}^{\prime} & \text { if }(i, k)=\left(j_{1}, N\right), \\ \lambda[1]_{i}^{(k)}+x_{i} & \text { if } k=N \text { and } \\ & j_{1}-1 \geq i>j_{2}, \\ \lambda[1]_{i}^{(k)}-y_{t-1} & \text { if }(i, k)=\left(t-1, N^{\prime}\right), \\ \lambda[1]_{i}^{(k)}+y_{t-1}-\left(x_{j_{2}+1}+\ldots+x_{j_{1}-1}+x_{j_{1}}^{\prime}\right) & \text { if }(i, k)=\left(j_{2}, N\right), \\ \lambda[1]_{i}^{(k)} & \text { if otherwise. }\end{cases}
$$

By $(* *)$, we have:

$$
\lambda[1]_{j_{1}}^{(N)}-i+s_{N}-\frac{N e}{l}>\lambda[1]_{t-1}^{\left(N^{\prime}\right)}-(t-1)-y_{t-1}+s_{N^{\prime}}-\frac{N^{\prime} e}{l} .
$$

Moreover, we have $\lambda[1]_{j_{1}}^{(N)}+x_{j_{1}}^{\prime}=\lambda[1]_{j_{1}-1}^{(N)}+1$ and $\lambda[1]_{i}^{(N)}+x_{i}=\lambda[1]_{i-1}^{(N)}+1$ for all $i$ such that $j_{1}-1 \geq i \geq a$. Thus, for all $i$ such that $j_{1}-1 \geq i \geq j_{2}$, by $(* *)$, we obtain:

$$
\lambda[1]_{i}^{(N)}-i+s_{N}-\frac{N e}{l}>\lambda[1]_{t-1}^{\left(N^{\prime}\right)}-(t-1)+x_{i+1}+\ldots+x_{j_{1}-1}+x_{j_{1}}^{\prime}-y_{t-1}+s_{N^{\prime}}-\frac{N^{\prime} e}{l} .
$$

Hence, by Proposition 4.3 we conclude that:

$$
\underline{\lambda}[2] \prec \underline{\lambda}[1] .
$$


Keeping the above figure, $\underline{\lambda}[2]$ is as follows.

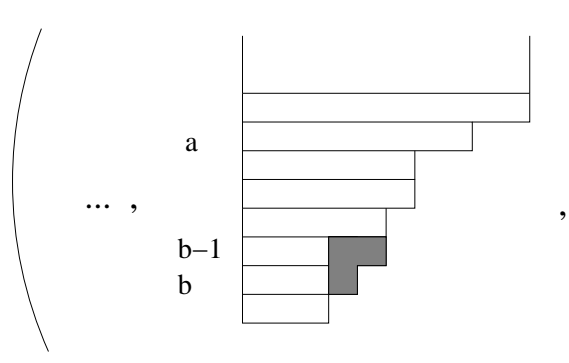

$\mathrm{N}$

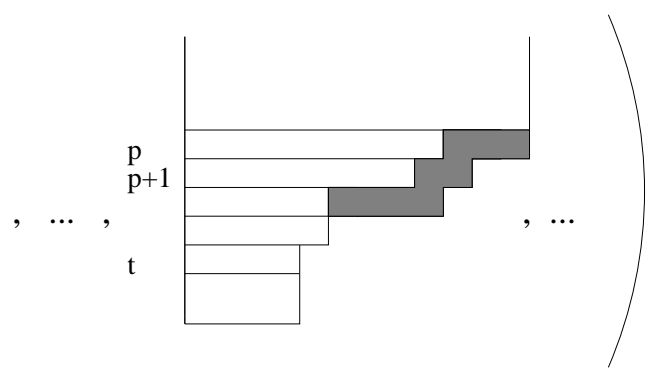

$\mathrm{N}^{\prime}$

Continuing in this way, we obtain the $l$-composition $\underline{\lambda}[p-t+1]=\underline{\lambda}$ :

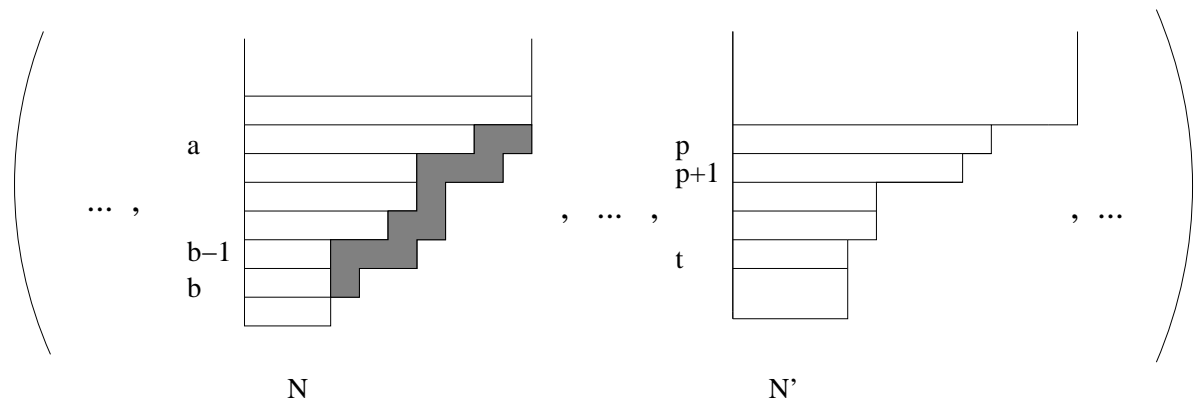

We have :

$$
\underline{\lambda} \prec \underline{\lambda}^{\prime} .
$$

Thus, we conclude that:

$$
a_{\mathbf{s}_{l}}(\underline{\lambda})<a_{\mathbf{s}_{l}}\left(\underline{\lambda^{\prime}}\right) .
$$

Now all the ordered monomials $u_{\underline{p}}$ which appear in $\overline{u_{\underline{k}}}$ are obtained recursively by using the relations $(R 1)-(R \overline{4})$. Hence by induction, we obtain that there exists $\beta$ such that:

$$
\overline{u_{\underline{k}}}=\beta u_{\underline{k}}+\text { sum of ordered monomials } u_{\underline{r}} \text { with } a\left(u_{\underline{r}}\right)>a\left(u_{\underline{k}}\right),
$$

By [U] Remark 3.24], we have $\beta=1$. This concludes the proof of the proposition.

\section{C Consequences}

The result of the previous section leads to the following theorem:

Theorem 4.5 Let $\mathbf{s}_{l} \in \mathbb{Z}^{n}$ and let $M_{\mathbf{s}_{l}}:=U_{q}\left(\widehat{\mathfrak{s l _ { e }}}\right) \cdot\left|\underline{\emptyset}, \mathbf{s}_{l}\right\rangle \subset \mathcal{F}_{e, \mathbf{s}_{l}}$. Let

$$
\left\{G\left(\underline{\mu}, \mathbf{s}_{l}\right) \mid \underline{\mu} \in \Lambda_{e ; \mathbf{s}_{l}}^{n}, n \in \mathbb{N}\right\}
$$

be the canonical basis elements. Then, for all $n \in \mathbb{N}$ and $\underline{\mu} \in \Lambda_{e ; \mathbf{s}_{l}}^{n}$, we have:

$$
G\left(\underline{\mu}, \mathbf{s}_{l}\right)=\left|\underline{\mu}, \mathbf{s}_{l}\right\rangle+\sum_{\underline{\lambda} \in \Pi_{l}^{n}, a_{\mathbf{s}_{l}}(\underline{\lambda})>a_{\mathbf{s}_{l}}(\underline{\mu})} d_{\underline{\lambda}, \underline{\mu}}(q)\left|\underline{\lambda}, \mathbf{s}_{l}\right\rangle,
$$

where $d_{\underline{\lambda}, \underline{\mu}}(q) \in q \mathbb{C}[q]$. 
Proof. By Proposition 4.4 we have for all $\underline{\lambda} \in \Pi_{l}^{n}$ :

$$
\overline{\left|\underline{\lambda}, \mathbf{s}_{l}\right\rangle}=\left|\underline{\lambda}, \mathbf{s}_{l}\right\rangle+\operatorname{sum} \text { of }\left|\underline{\mu}, \mathbf{s}_{l}\right\rangle \text { with } a_{\mathbf{s}_{l}}(\underline{\mu})>a_{\mathbf{s}_{l}}(\underline{\lambda}) .
$$

Assume that $\underline{\lambda} \in \Lambda_{e ; \mathbf{s}_{l}}^{n}$ and that $\underline{\mu}$ is one of the minimal $l$-partitions with respect to $a_{\mathbf{s}_{l}}$ which appears in $G\left(\underline{\lambda}, \mathbf{s}_{l}\right)$. Then the characterization of the canonical basis in Theorem 3.2 immediately implies that $\underline{\lambda}=\underline{\mu}$.

Now, we give the consequences on the decomposition matrices of Ariki-Koike algebras. By Ariki's theorem, we obtain the following result. Note that this generalizes and gives a new proof of the main result of Jp where the case $0 \leq s_{1} \leq \ldots \leq s_{l}<e$ was considered (but the proof of [Jp] gave an explicit construction of the canonical basis elements). Note also that it provides an interpretation of the parametrization by the Kleshchev $l$-partitions by using $a$-values.

Let $\mathcal{H}_{\mathbb{C}, n}$ be the Ariki-Koike algebra over $\mathbb{C}$ with the following choice of parameters:

$$
\begin{aligned}
& x_{j}=\eta_{e}^{v_{j}} \text { for } j=1, \ldots, l, \\
& x=\eta_{e},
\end{aligned}
$$

where $0 \leq v_{1} \leq \ldots \leq v_{l}<e$.

Let $\mathbf{s}_{l}=\left(s_{1}, \ldots, s_{l}\right) \in \mathbb{Z}^{l}$ be such that $s_{i}=v_{i}(\bmod e)$ for $i=1, \ldots, l$ and let $\mathcal{H}_{\mathbb{C}(y), n}^{\mathbf{s}_{l}}$ be the Ariki-Koike algebra over $\mathbb{C}(y)$ with the following parameters:

$$
\begin{aligned}
& u_{j}=y^{l m^{(j)}} \eta_{l}^{j-1} \text { for } j=1, \ldots, l, \\
& v=y^{l}
\end{aligned}
$$

where $m^{(j)}=s_{j}-\frac{(j-1) e}{l}+\alpha e$ for $j=1, \ldots, l$ and where $\alpha$ is a positive integer such that $m^{(j)}>0$ for $j=1, \ldots, l$. Then the specialisation $\theta: \mathbb{C}\left[y, y^{-1}\right] \rightarrow \mathbb{C}$ which sends $y$ to $\exp \left(\frac{2 i \pi}{l e}\right)$ induces a decomposition map:

$$
d: R_{0}\left(\mathcal{H}_{K, n}^{\mathbf{s}_{l}}\right) \rightarrow R_{0}\left(\mathcal{H}_{\mathbb{C}, n}\right) .
$$

Theorem 4.6 For each $M \in \operatorname{Irr}\left(\mathcal{H}_{\mathbb{C}, n}\right)$, there exists a unique simple $\mathcal{H}_{\mathbb{C}(y), n^{-}}^{\mathbf{s}_{l}}$ module $V_{M}$ such that the following two conditions hold:

- $d_{V_{M}, M}=1$,

- if there exists $W \in \mathcal{H}_{\mathbb{C}(y), n}^{\mathbf{s}_{l}}$ such that $d_{W, M} \neq 0$ then $a(W)>a\left(V_{M}\right)$.

Moreover the application which sends $M$ to $V_{M}$ is injective. As a consequence the associated decomposition matrix is unitriangular and the following set is in natural bijection with $\operatorname{Irr}\left(\mathcal{H}_{\mathbb{C}, n}\right)$ :

$$
\mathcal{B}_{\mathbf{s}_{l}}=\left\{V_{M} \mid M \in \operatorname{Irr}\left(\mathcal{H}_{\mathbb{C}, n}\right)\right\} .
$$

Finally, we have:

$$
\mathcal{B}_{\mathbf{s}_{l}}=\left\{S_{\overline{\mathbb{C}}(y)}^{\underline{\lambda}} \mid \underline{\lambda} \in \Lambda_{e ; \mathbf{s}_{l}}^{n}\right\} .
$$


Example 4.7 We keep the example [3.6, the decomposition matrices of ArikiKoike algebras can be computed using the algorithm described in [Ja]. For $l=2$, $e=4, v_{1}=0, v_{2}=1$, we obtain the following matrices which are written using the different labelings of the above theorem. The rows of the matrices are indexed by the l-partitions of rank 4 , we also give the associated a-values.

- When $s_{1}=0$ and $s_{2}=1$, the simple $\mathcal{H}_{\mathbb{C}, n}$-modules may be labeled by $\Lambda_{4,(0,1)}^{4}$ :

$$
\left.\begin{array}{cc|ccccccccccccc}
((4), \emptyset) & 0 & 1 & . & . & . & . & . & . & . & . & . & . & . & . \\
((3),(1)) & 1 & . & 1 & . & . & . & . & . & . & . & . & . & . & . \\
(\emptyset,(4)) & 1 & . & . & 1 & . & . & . & . & . & . & . & . & . & . \\
((3,1), \emptyset) & 1 & 1 & . & . & 1 & . & . & . & . & . & . & . & . & . \\
((2),(2)) & 2 & . & 1 & . & . & 1 & . & . & . & . & . & . & . & . \\
((2,2), \emptyset) & 2 & . & . & . & . & . & 1 & . & . & . & . & . & . & . \\
((1),(3)) & 2 & 1 & . & 1 & . & . & . & 1 & . & . & . & . & . & . \\
((2,1),(1)) & 3 & . & . & . & . & . & . & . & . & . & . & . & . & . \\
((2,1,1), \emptyset) & 4 & . & . & . & 1 & . & . & . & 1 & . & . & . & . & . \\
((2),(1,1)) & 4 & . & . & . & . & . & . & . & . & 1 & . & . & . & . \\
((1,1),(2)) & 4 & 1 & . & . & 1 & . & . & . & . & . & 1 & . & . & . \\
((1),(2,1)) & 5 & . & . & . & . & . & . & . & . & . & . & 1 & . & . \\
((1,1),(1,1)) & 6 & . & . & . & . & . & . & . & . & . & . & . & 1 & . \\
(\emptyset,(3,1)) & 4 & . & . & 1 & . & . & . & . & . & . & . & . & . & 1 \\
((1,1,1),(1)) & 6 & . & . & . & 1 & . & . & . & . & . & . & . & . & . \\
(\emptyset,(2,2)) & 6 & . & . & . & . & 1 & . & . & . & 1 & . & 1 & . & . \\
((1,1,1,1), \emptyset) & 9 & . & . & . & . & . & . & . & . & . & . & . & . & . \\
(\emptyset,(2,1,1)) & 9 & . & . & . & . & . & . & 1 & . & 1 & . & . & . & . \\
((1),(1,1,1)) & 9 & . & . & . & . & . & . & . & . & . & . & 1 & . & . \\
(\emptyset,(1,1,1,1)) & 16 & . & . & . & . & . & . & . & . & . & . & . & . & 1 \\
(16 & & & . & . & . & 1 & . & .
\end{array}\right)
$$

- When $s_{1}=4$ and $s_{2}=1$, the simple $\mathcal{H}_{\mathbb{C}, n}$-modules may be labeled by $\Lambda_{4,(4,1)}^{4}$ : 


\begin{tabular}{|c|c|c|c|c|c|c|c|c|c|c|c|c|c|}
\hline$((4), \emptyset)$ & 0 & 1 & & & & & & & & & & & \\
\hline$((3,1), \emptyset)$ & 1 & 1 & 1 & & & & & & & & & & . \\
\hline$((2,2), \emptyset)$ & 2 & & & & & & & & 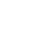 & & & . & \\
\hline$((2,1,1), \emptyset)$ & 3 & & 1 & & 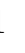 & & & & 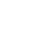 & . & & . & . \\
\hline$((3),(1))$ & 5 & & & & & & & & 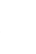 & & & . & . \\
\hline$((2,1),(1))$ & 6 & & & & & & & & . & & & . & . \\
\hline$((1,1,1),(1))$ & 8 & & 1 & & L & & & & 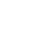 & . & & & . \\
\hline$((2),(2))$ & 9 & & & & & & & & L & & & . & \\
\hline$((1,1),(2))$ & 10 & 1 & 1 & & & & & & & 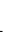 & & . & . \\
\hline$((2),(1,1))$ & 12 & & & & & & & & & & 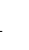 & & . \\
\hline$((1),(3))$ & 12 & 1 & & & & & & & & 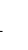 & & 1 & \\
\hline$((1,1),(1,1))$ & 13 & & & & & & & 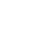 & 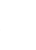 & & & . & 1 \\
\hline$((1),(2,1))$ & 16 & & • & & • & & & 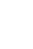 & 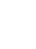 & & & . & \\
\hline$((1,1,1,1), \emptyset)$ & 6 & & & & 1 & & & 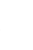 & 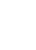 & s. & ? & . & . \\
\hline$(\emptyset,(4))$ & 14 & & & & & & & 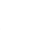 & 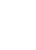 & & & 1 & . \\
\hline$(\emptyset,(3,1))$ & 19 & & & & & & & & & 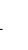 & & 1 & . \\
\hline$(\emptyset,(2,2))$ & 22 & & & & & & & & L & & & & . \\
\hline$(\emptyset,(2,1,1))$ & 25 & & & & & & & L & & 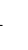 & & & . \\
\hline$((1),(1,1,1))$ & 21 & & & & & & & . & 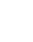 & & & . & 1 \\
\hline$(\emptyset,(1,1,1,1))$ & 32 & & & & & & & 1 & & & & . & . \\
\hline
\end{tabular}

- When $s_{1}=0$ and $s_{2}=5$, the simple $\mathcal{H}_{\mathbb{C}, n}$-modules may be labeled by $\Lambda_{4,(0,5)}^{4}$ :

\begin{tabular}{|c|c|c|c|c|c|c|c|c|c|c|c|c|}
\hline$(\emptyset,(4))$ & 0 & 1 & & & . & & . & & & & & \\
\hline$(\emptyset,(3,1))$ & 1 & 1 & 1 & & . & - & . & & . & . & & \\
\hline$(\emptyset,(2,2))$ & 2 & & . & 1 & . & - & . & & . & . & & . \\
\hline$((1),(3))$ & 3 & 1 & 1 & . & 1 & . & . & & . & . & . & \\
\hline$(\emptyset,(2,1,1))$ & 3 & & 1 & 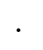 & . & 1 & . & & . & & . & \\
\hline$((1),(2,1))$ & 4 & & & . & . & . & 1 & & 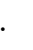 & . & 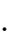 & \\
\hline$((2),(2))$ & 5 & & 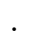 & 1 & . & . & . & 1 & . & . & & \\
\hline$((1),(1,1,1))$ & 6 & & . & . & . & . & . & & 1 & . & & \\
\hline$((2),(1,1))$ & 6 & & . & . & . & . & 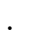 & & . & . & & \\
\hline$((1,1),(2))$ & 8 & & 1 & 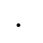 & 1 & 1 & 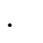 & & & 1 & & \\
\hline$((1,1),(1,1))$ & 9 & & & . & $\cdot$ & . & . & & 1 & & 1 & \\
\hline$((2,1),(1))$ & 10 & & & ${ }^{\circ}$ & . & . & . & & 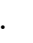 & . & & \\
\hline$((1,1,1),(1))$ & 15 & & & . & . & 1 & . & & . & 1 & & \\
\hline$(\emptyset,(1,1,1,1))$ & 6 & $\cdot$ & & . & 1 & . & . & . & . & . & . & \\
\hline$((4), \emptyset)$ & 6 & & & . & . & & . & 1 & . & . & . & \\
\hline$((3),(1))$ & 6 & & & . & . & 1 & . & & . & . & & \\
\hline$((3,1), \emptyset)$ & 11 & & & . & 1 & . & . & & . & 1 & & \\
\hline$((2,2), \emptyset)$ & 14 & & & . & . & . & . & & • & & 1 & \\
\hline$((2,1,1), \emptyset)$ & 17 & & & . & . & . & . & & $\cdot$ & 1 & 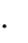 & \\
\hline$((1,1,1,1), \emptyset)$ & 24 & & & . & . & . & . & . & $\cdot$ & & & \\
\hline
\end{tabular}




\section{References}

[As] S. Ariki, On the semi-simplicity of the Hecke algebra of $(\mathbf{Z} / r \mathbf{Z})$ ? $S_{n}$. J. Algebra 169, no. 1 (1994): 216-225.

[Ad] S. Ariki, On the decomposition numbers of the Hecke algebra of $G(m, 1, n)$. J. Math. Kyoto Univ., 36 (1996): 789-808.

[Ac] S. Ariki, On the classification of simple modules for Cyclotomic Hecke algebras of type $G(m, 1, n)$ and Kleshchev multipartitions. Osaka J.Math., 38 (2001): 827-837.

[Ab] S. Ariki, Representations of quantum algebras and combinatorics of Young tableaux. Univ. Lecture Series, 26 (2002), AMS.

[AK] S. Ariki, K. Koike, A Hecke algebra of $(\mathbb{Z} / r \mathbb{Z})$ ? $\mathfrak{S}_{n}$ and construction of irreducible representations. Adv. Math., 106 (1994): 216-243.

[BM] M. Broué, G. Malle, Zyklotomische Heckealgebren. Astérisque, 212 (1993): 119-189.

[DJM] R. Dipper, G. James, A. Mathas, Cyclotomic q-Schur algebras. Math. Z., 229 no. 3 (1998): 385-416.

[DM] R. Dipper, A. Mathas, Morita equivalences of Ariki-Koike algebras, Math.Z., 240 no. 3 (2003): 579-610.

[FL] O. Foda, B. Leclerc, M. Okado, J-Y Thibon, T. Welsh, Branching functions of $A_{n-1}^{(1)}$ and Jantzen-Seitz problem for Ariki-Koike algebras. Adv. Math., 141 no. 2 (1999), 322-365.

[GL] J. Graham, G. Lehrer, Cellular algebras. Invent. Math., 123 (1996): 1-34.

[Gm] M. Geck, Representations of Hecke algebras at roots of unity. Séminaire Bourbaki. Vol. 1997/98. Astérisque 252 (1993): 33-55.

[G] M. Geck, Kazhdan-Lusztig cells and decompositions numbers, Representation theory, 2 (1998): 264-277.

[GIM] M. Geck, L. Iancu, G. Malle, Weights of Markov traces and generic degrees. Indag. Math., 11 (2000): 379-397.

[GR] M. Geck, R. Rouquier, Filtrations on projective modules for Iwahori-Hecke algebras. Modular representation theory of finite groups (Charlottesville, VA, 1998), de Gruyter, Berlin (2001): 211-221.

[Jp] N. Jacon, On the parametrization of the simple modules for Ariki-Koike algebras, J. Math. Kyoto Univ, 44 no. 4 (2004): 729-767.

[Ja] N. Jacon, An algorithm for the computation of the decomposition matrices for Ariki-Koike algebras, J. Algebra (to appear).

[JM] M. Jimbo, K. Misra, T. Miwa, M. Okado, Combinatorics of representations of $\mathcal{U}_{q}(\widehat{s l}(n))$ at $q=0$. Commun. Math. Phys., 136 (1991): 543-566. 
[LT] B. Leclerc, J-Y Thibon, Canonical bases of q-deformed Fock spaces. Int. Math. Res. Notices, 9 (1996): 447-456.

[Ms] A. Mathas Simple modules of Ariki-Koike algebras, in Group representations: cohomology, group actions and topology, Proc. Sym. Pure Math., 63 (1998): 383-396.

[Ma] A. Mathas The representation theory of the Ariki-Koike and cyclotomic $q$-Schur algebras, Representation theory of algebraic groups and quantum groups, Adv. Studies Pure Math. (2004): 261-320.

[TU] H. Takemura, D. Uglov, Representations of the quantum toroidal algebra on highest weight modules of the quantum affine algebra of type $\mathfrak{g l}_{N}$. Publ. Res. Inst. Math. Sci. 35, No.3 (1999): 407-450.

[U] D. Uglov, Canonical bases of higher-level q-deformed Fock spaces and Kazhdan-Lusztig polynomials, Kashiwara, Masaki (ed.) et al., Boston: Birkhäuser. Prog. Math. 191 (2000): 249-299.

[Us] D. Uglov, Canonical base of higher-level q-deformed Fock spaces (short version of [U] math.QA/9901032 